\title{
A Library of Prompt Detonation Reaction Zone Data
}

\author{
P. Clark Souers
}

June 1998

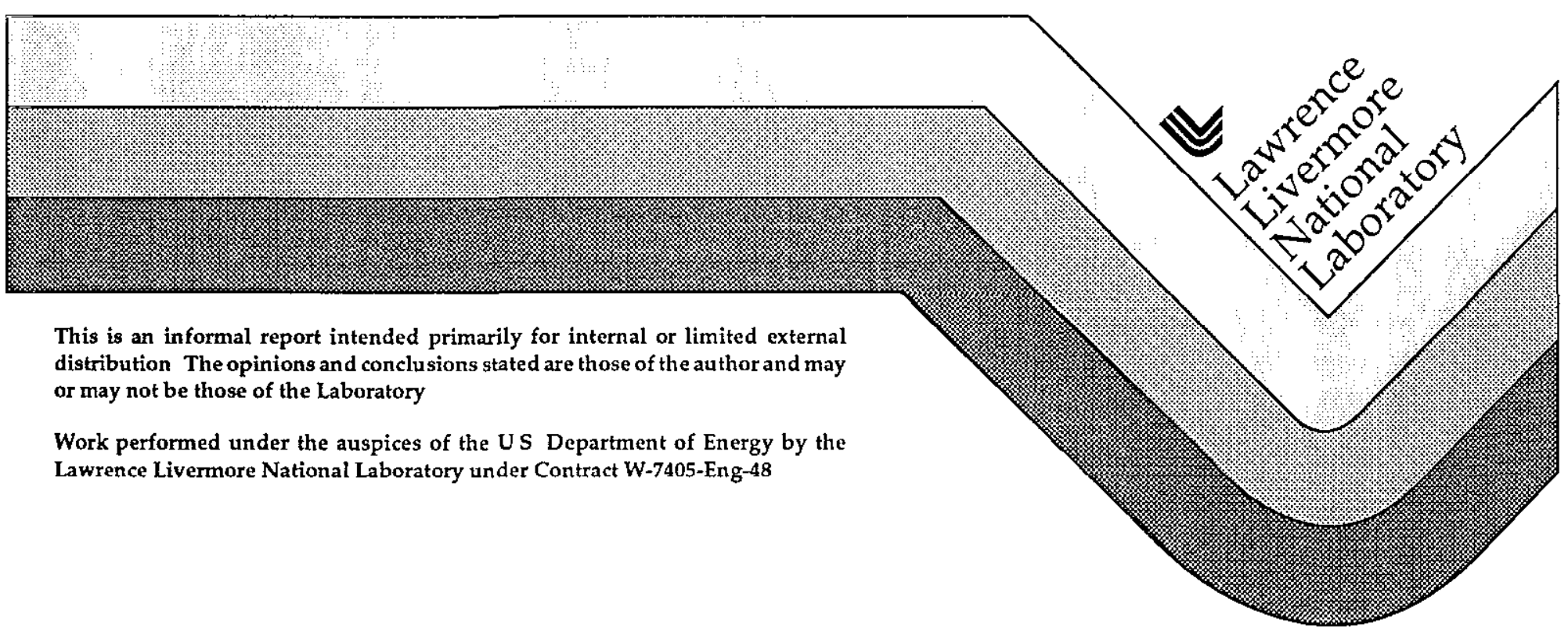




\section{DISCLAIMER}

This document was prepared as an account of work sponsored by an agency of the United States Government. Neither the United States Government nor the University of California nor any of their employees, makes any warranty, express or implied, or assumes any legal liability or responsibility for the accuracy, completeness, or usefulness of any information, apparatus, product, or process disclosed, or represents that its use would not infringe privately owned rights. Reference herein to any specific commercial product, process, or service by trade name, trademark, manufacturer, or otherwise, does not necessarily constitute or imply its endorsement, recommendation, or favoring by the United States Government or the University of California. The views and opinions of authors expressed herein do not necessarily state or reflect those of the United States Government or the University of California, and shall not be used for advertising or product endorsement purposes.

This report has been reproduced directly from the best available copy.

Available to DOE and DOE contractors from the Office of Scientific and Technical Information

P.O. Box 62, Oak Ridge, TN 37831

Prices available from (615) 576-8401, FTS 626-8401

Available to the public from the

National Technical Information Service

U.S. Department of Commerce

5285 Port Royal Rd.,

Springfield, VA 22161 


\begin{abstract}
Tables are given listing literature data that allows calculation of sonic reaction zones at or near steadystate for promptly-detonating explosive cylinders The data covers homogeneous, heterogeneous, composite, inorganic and binary explosives and allows comparison across the entire explosive field Table 1 lists detonation front curvatures Table 2 lists Size Effect data, ie the change of detonation velocity with cylinder radius Table 3 lists failure radii and detonation velocities Table 4 lists explosive compositions A total of 51 references dating back into the 1950's are given Calculated reaction zones, radii of curvature and growth rate coefficients are listed
\end{abstract}

\title{
Table of Contents
}

I Description of the Tables

Table 1 Detonation Front Curvature

I-3

Table 2 Size Effect

I-4

Table 3 Failure Radius

$\mathrm{I}-4$

Table 4 Compositions of Explosives

I-5

Table References

I-5

II Comparing the Data

II-1

Tables

Table 1 Detonation Front Curvature

$T-1$

Table 2A Size Effect Homogeneous and Heterogeneous T-5

Table 2B Size Effect Composite and Inorganic $\quad$ T-12

Table 3 Failure Radius

Table 4 Compositions of Explosives

$\mathrm{T}-18$

$\mathrm{T}-21$ 


\section{Description of the Tables}

The intent is to collect steady state, prompt detonation data that pertains to calcuation of the average sonic reaction zone length, ie the distance behind the detonation front that furnishes chemical energy to push the fiont We here present results on the detonation front curvature in cylinders and the Size Effect, ie the change of detonation velocity with cylinder radius The data is listed plus the calculated parameters that constitute the input to Larry Fried's upcoming LLNL code KINETIC CHEETAH

Table 1: Detonation Front Curvature

A collection of cylinder detonation front curvature data, as obtained from streak camera measurements, follows, necessarily compressed from the original data files The data is divided into sections with attempts to create families The 92 5-97 $5 \mathrm{wt} \%$ TATB family is the only one where explosives from different sources are collected, because of the general agreement between samples The ANFO and PBXW-111 families are separated not only as to where they came from but also whether they are confined or unconfined In each family, the data is ordered from small to large radii

The sections may be seen to fall into larger groups Most of the table is made up of "quadratic" curvatures, ie those where the radius-squared makes up half or more of the lag and where the curves are smooth across the entire diameter The top sections are homogeneous and heterogenerous explosives, the bottom sections are composites Near the bottom comes the small set of "non-quadratic" curves, which have steep edge lags and ragged centers These occur only for explosives with short reaction zones We next have the extreme nonquadratic curves where defects of the order of $1 / 10$ th the cylinder radius have severely broken up the front but steep edge effects are still clearly visible Finally, we have three quadratic slabs with a square cross section

The columns contain the following

1) the explosive name

2) initial density, $\rho_{0}$

3) initial outer radius of the explosive, $R_{0}$, and inner radius of a metal sleeve if present, for a cylinder For the slabs, $R_{0}$ is the half-width

4) measured edge lag, $L_{o}$, where the data was taken all the way out to the edge

5) calculated reaction zone lengths, $\left\langle x_{\mathrm{e}}\right\rangle$ in $\mathrm{mm}$ using the theory from $\mathrm{P} C$ Souers and $\mathrm{R}$ Garza, "Kinetic Information from Detonation. Front Curvature, " Eleventh International

Detonation Symposium, submitted

6) measured detonation velocity, $U_{s}$, for the radius $R_{0}$

7) infinite-radius/infinite containment detonation velocity, $\mathrm{D}$, obtained by the extrapolation of the inverse radius to zero 
8) lag-fit coefficient of the radius-squared, A, which describes the sideways energy flow across the entire cylinder

9) lag-fit coefficient of the radius to the sixth power, $B$, which describes the effects of the edge Note A quadratic curve is fit by the lag equation $\mathrm{L}=\mathrm{AR}^{2}+\mathrm{BR}^{6}$, a non-quadratic curve is fit by $\mathrm{BR}^{6}$

10) measured edge angle, where the error is generally $\pm 5^{\circ}$ to $\pm 10^{\circ}$

11) calculated edge angle, obtained from the derivitive of the lag fit equation

12) growth rate coefficient, $G$, in the $\mathrm{GP}^{2}(1-\mathrm{F})$ overall explosive reaction rate

13) radius of curvature on the cylinder axis, $\mathrm{R}_{\text {cur }}$, as obtained directly from the curvature fit coefficient $\mathrm{A}$

14) wall confinement material, if any

15) wall confinement thickness in $\mathrm{mm}$

16) energy conductivity, $\mathrm{K}$ (in $\mathrm{MW} / \mathrm{mm}^{2}$ ), as calculated from the quadratic coefficient, A, and which seeks to describe transverse energy flow across the cylinder

17) length of cylinder in $\mathrm{mm}$

18) reference

The material of confinement is listed where known, even though its effect might have been negligible The abbreviations are

$\mathrm{Br}$ brass, $856 \mathrm{~g} / \mathrm{cc}$

$\mathrm{Cb}$ cardboard, $05 \mathrm{~g} / \mathrm{cc}$

$\mathrm{Cu}$ copper, $893 \mathrm{~g} / \mathrm{cc}$

PVC plastic, $15 \mathrm{~g} / \mathrm{cc}$

Py pyrex glass, $22 \mathrm{~g} / \mathrm{cc}$

SS stainless steel, $78 \mathrm{~g} / \mathrm{cc}$
Some explosive families have been studied in different places The abbreviations for these are

1) $P B X N-111 N=U S$ Navy, $A=$ Australia

1) $\mathrm{ANFO} \mathrm{NM}=$ New Mexico, $\mathrm{SW}=$ Sweden

Table 2. Size Effect

The Size Effect is the change of detonation velocity with cylinder radius The data listed is

1) name, density, pre-pressed explosive particle size and reference

2) radius, $R_{0}$, in $\mathrm{mm}$

3) measured detonation velocity in $\mathrm{mm} / \mu \mathrm{s}$ The first entry opposite $R_{0}=\infty$ is the extrapolated infinite radius detonation velocity

4) calculated reaction zone length, $\left\langle x_{e}\right\rangle$ in $\mathrm{mm}$

5) the first number is the C-J pressure as calculated by CHEETAH It is used to calculate the growth rate coefficient, $G$, listed below it

6) radius of curvature, $R_{\text {cur }}$, on the cylinder axis as calculated from the detonation velocities

\section{Table 3. Failure Radius}

The failure radii of page 1 are mostly the subset of the Size Effect Listed are the following

1) density in $\mathrm{g} / \mathrm{cc}$ 
2) failure radius, $R_{f}(\mathrm{~mm})$ We use only data sets where it mentions that failure was observed The failure radius is the average of the smallest radius for which detonation was observed and the largest at which it was not The definition of failure is set by the researcher Campbell required propagation beyond four 50 mm-long rate sticks for PBX-9502, ${ }^{26}$ which is undoubtedly far stricter than the average definition All samples are unconfined unless noted

3) approximate detonation velocity at failure, $U_{s f}(\mathrm{~mm} / \mu \mathrm{s})$ This is usually the last measured value lowered to the nearest 01 $\mathrm{mm} / \mu \mathrm{s}$

4) infinite-radius/infinite containment detonation velocity, $D$, obtained by inversesquare extrapolation of the radius to zero

5) extrapolated sonic reaction zone length at failure, $<\mathrm{x}_{\mathrm{e}}>_{\mathrm{f}}(\mathrm{mm})$

6) calculated growth coefficient $G$

7) calculated radius of curvature on the axis, $\mathrm{R}_{\text {cur }}(\mathrm{mm})$

8) reference

Page 2 has the void volume fraction, $f_{v}$, and the inert material fraction $\mathrm{f}_{\mathrm{f}}$ and their sum

\section{Table 4. Compositions of Explosives}

All compositions are in wt \%

\section{ACKNOWLEDGEMENTS}

We wish to thank Jerry Forbes, Per-Anders Persson, John Bdzil, Damian Swift, Finn Ouchterlony and David Kennedy for their kind assistance in supplying curvature data from their laboratories This work was performed under the auspices of the U S Department of Energy by the Lawrence Livermore National Laboratory under contract number W-7405-ENG-48

\section{References}

1 LLNL Cylinder Test

2 John Bdzil, Los Alamos National Laboratory, Los Alamos, NM, private communication, 1996

3 J B Bdzil, $J$ Fluid Mech 108, 195-226 (1981)

4 R Engleke and J B Bdzil, Phys Fluids $26,1210(1983)$

5 Alan Collyer and Damian Swift, AWE, Aldermaston, Great Britain, private communication, 1996

$6 \mathrm{H}$ Moulard, J W Kury and A Delclos, "The Effect of RDX Particle Size on the Shock Sensitivity of Cast PBX Formulations," Proceedings Eighth Symposium (International) on Detonation, Albuquerque, NM, July 15-19, 1985, pp 902-913

7 H Moulard, "Particular Aspect of the Explosive Particle Size Effect on Shock Sensitivity of Cast PBX Formulations," Proceedings Ninth Symposium (Inter national) on Detonation, Portland, OR, August 28-September 1, 1989, pp 1824

$8 \mathrm{~J}$ W Forbes, E R. Lemar, G T Sutherland and R N Baker, Detonation Wave Curvature, Corner Turning and Unreacted Hugoniot of PBXN-111, Naval Surface Warfare Center Report NSWCDD/TR-92/164, Silver Spring, MD, 1992 PBXN-111 was previously called PBXW-115

9 David Kennedy, ICl Australia Operations, Kurri Kurri, New South Wales, Australia, private communications, 1995, 1997

10 U Nyberg, J Deng and L Chen, Matning av Detonationshastighet och Krokningsfront $i$ Samband med Brinnmodellutveckling for Emulsionssprangamne Kl, Swedish Rock 
Engineering Research, Stockholm, SveBeFo Report 6, 1995

11 J Deng, $S$ Nie and $L$ Chen, Deter mination of Burning Rate Parameters for an Emulsion Explosive, Swedish Rock Engineering Research, Stockholm, SveBeFo Report 17, 1995 Finn Ouchterlony kindly sent the Swedish Rock reports

12 Jaimin Lee, Detonation Shock Dynamics of Composite Energetic Materials, $\mathrm{PhD}$ Thesis, New Mexico Institute of Mining and Technology, Socorro, New Mexico, 1990 Per-Anders Persson kindly lent us a copy of the thesis

13 F Chaisse' and J N Oeconomos, "The Shape Analysis of a Steady Detonation Front in Right Circular Cylinders of High Density Explosive Some Theoretical and Numerical Aspects," Proceedings Tenth Symposium (International) on Detonation, Boston, MA, July 12-16, 1993, pp 50-57

14 E R Lamar and J W Forbes, "Detonation Wave Curvature of Cast Comp B and PBXN-111," American Physical Society Topical Group on Shock Compression, Colorado Springs, CO, June 28-July 2, 1993, Proceedings, pp 1385-1388

$15 \mathrm{~J}$ W Forbes and E R Lamar, "Detonation Wave Velocity and Curvature of Brass Encased PBXN-111," American Physical Society Topical Group on Shock Compression, Seattle, WA, August 13-18, 1995, Proceedings, pp 787-790

16 E R Lemar, J W Forbes and G $T$ Sutherland, "Detonation Wave Velocity and Curvature of IRX-4 and PBXN-110," American Physical Society Topical Group on Shock Compression, Seattle, WA, August 13-18, 1995, Proceedings, pp 791-794

17 G T Sutherland, E R Lamar, J W Forbes, E Anderson, P Miller, K D Ashwell, R N Baker, and T P Liddiard, "Shock Wave and Detonation Wave Response of Selected HMX Based Research Explosives with HTPB Binder Systems," American Physical Society Topical Group on Shock Compression, Colorado Springs, CO , June 28-July 2, 1993, Proceedings, pp 1413-1416
18 W H Wilson, J W Forbes, $\mathrm{P}$ K Gustavson, E R Lemar and G $T$ Sutherland, "Detonation Properties of the Non-Ideal Explosive PBXW-123," American Physical Society Topical Group on Shock Compression, Seattle, WA, August 13-18, 1995, Proceedings, pp 795-798

19 J O Erkman and D Price, Comparison of Curvature of Detonation Front in AP with that found in some Conventional Explosives, U S Naval Ordnance Laboratory, White Oak, MD, report NOLTR 69-235 (1970) The listed lag axis has been multiplied by 100

$20 \mathrm{M} \mathrm{J}$ Urizar, E James, Jr and L C Smith, "The Detonation Velocity of Pressed TNT," Third Symposium on Detonation, Princeton, NJ, September 26-28, 1960, pp 327-356

21 E A Igel and L B Seely, Jr, "The Detonation Behavior of Liquid TNT," Second ONR Symposium on Detonation, White Oak, MD, February 11, 1955, pp 439-453

22 LASL Explosive Property Data, T R Gibbs and A Popolato, ed (University of California Press, Berkeley, 1980), section following $\mathrm{p} 236$ The XTX-8003 "diameters" on p 249 are really radii (see ref 27)

23 M E Malin, A W Campbell and G W Mautz, "Particle Size Effects in One- and Two-Component Explosives," Second ONR Symposium on Detonation, White Oak, MD, February 11, 1955, pp 478-493

24 L B Chapman, "NTO Development at Los Alamos," Proceedings Ninth Symposium (International) on Detonation, Portland, OR, August 28-Septmeber 1, 1989, pp 1001-1006

25 P Gimenez, $\mathrm{P}$ Chabin, J Mala and C Spyckerelle, "An Extensive Experimental Study of Pressed NTO," Proceedings Tenth Inter national Detonation Symposium, Boston, MA, July 12-16, 1993, pp 276-283

26 A W Campbell, "Diameter Effect and Failure Diameter of a TATB-Based Explosive," Propellants, Explosives, Pyrotechnics, 9, 183-187 (1984) 
27 A W Campbell and R Engelke, "The Diameter Effect in High-Density Heterogeneous Explosives," Proceedings Sixth Symposium (International) on Detonation, Coronado, CA, August 24-27, 1976, pp 642- 652

28 R H Dinegar, "Detonation Velocity of PETN in Small Confined Cylindrical Charges," Propellants, Explosives, Pyrotechnics, 1, 97-100 (1976)

29 C D Hutchinson, G C W Foan, $H$ R Lawn and A G Jones, "Initiation and Detonation Properties of the Insensitive High Explosive TATB/Kel-F 800 95/5", Proceedings Ninth Symposium (International) on Detonation, Portland, OR, August 28-Septmeber 1, 1989, pp 123132

30 M A Cook, E B Mayfield and W S Partridge, "Reaction Rates of Ammonium Nitrate in Detonation," $J$ Phys Chem 59, 675-680 (1955)

31 P C Souers, D B Larson and C M Tarver, Performance Calculations on the ANFO Explosive RX-HD, LLNL report UCRL-ID-1 18969 (1995) The RX-HD was from Dyno Nobel

32 F Helm, M Finger, B Hayes, E Lee, H Cheung and J Walton, High Explosive Characterization for the Dice Throw Event, LLNL report UCRL-52042 (1976)

33 Hino and Yokogawa, data scanned from $W$ E Gordon, "Detonation Limits in Condensed High Explosives," Proceedings Four th Symposium (International) on Detonation, White Oak, MD, October 1215, 1965, pp 179-197, see Fig 1c

34 R H Hales, Final Report for the QM-100R Program, IRECO Inc, West Jordan UT, submitted to New Mexico Engineering Institute, Albuquerque, NM, March 19, 1993

35 QM-100 obtained with some data from Clark Bonner of IRECO Inc, West Jordan UT, 1993 Also supporting data from R Simpson, LLNL, in conjunction with the QM-100 cylinder shots at LLNL, 1992
36 M A Cook and W S Partridge, "Detonation Properties and Reaction Kinetics of 2,4-Dinitrotoluene," $J$ Phys Chem, 59, 673-675 (1955)

37 L N Stesik and L N Akimova, "An Indirect Method of Estimating the Reaction

Zone Width of a Detonation Wave," Russ $J$ Phys Chem, 33, 148- 151 (1959)

38 M A Cook, R T Keyes, W S Partridge and W O Ursenback, "Velocity-Diameter Curves, Velocity Transients and Reaction Rates in PETN, RDX, EDNA and Tetryl," $J$ Amer Chem Soc , 79, 32-37 (1957)

39 P Deneuville, C Gaudin, Y de Longueville and J Mala, "Comparison of TATB and DINGU Explosive Properties," Proceedings Seventh Symposium (Inter national) on Detonation, Annapolis, MD, June 16-19, 1981, pp 540-546

40 Y De Longueville, A Delclos, C Gaudin and J Mala, "Influence of Inert Binders on Detonation Properties of Cast-Cured PBX," Proceedings Seventh Symposium (International) on Detonation, Annapolis, MD, June 16-19, 1981, pp 560-565

41 A W Campbell, H L Flaugh, A Popolato and J B. Ramsay, "Customized Explosives Based on Plastic-Bonded Mixtures to TATB and HMX," Proceedings Seventh Symposium (International) on Detonation, Annapolis, MD, June 16-19, 1981, pp 566-574

42 V M Titov, V V Sil'vestrov, V V Kravtsov and I A Stadnitschenko, "Investigation of Some Cast TNT Properties at Low Temperatures," Proceedings Sixth Symposium (International) on Detonation, Coronado, CA, August 24-27, 1976, pp 36-45

43 R J Spear and M G Wolfson, Determination of Detonation Parameters of Booster Explosives at Small Diameter Charges, DSTO Materials Research Laboratory MRL-TR-89-45, Cordite Avenue, Maribyrnong, Victoria 3032, Australia (1990), from Defense Center Information Center, Ft Belvoir, VA, ADA220 352, Ar-006-287 (1990) 
44 M E Evans, B O Reese, L B Seely and E L Lee, "Shock Initiation of Low-Density Pressings of Ammonium Perchlorate," Proceedings Fourth Sympositum (International) on Detonation, White Oak, MD, October 12-15, 1965, pp 359-371

45 A R Clairmont, Jr, I Jaffe and D Price, The Detonation Behavior of Ammonium Perchlorate as a Function of Charge Density and Diameter, U S Naval Ordnance Labor atory NOLTR-67-71, White Oak, MD (1967) p 24

46 M L Pandow, $\mathrm{K}$ F Ockert and $\mathrm{H} \mathrm{M}$ Shuey, "Studies of the DiameterDependence of Detonation Velocity in Solid Composite Propellants," Proceedings Four th Symposium (International) on Detonation, White Oak, MD, October 1215, 1965, pp 96-101

47 D Price, A R Clairmont, Jr and J O Erkman, "Explosive Behavior of Aluminized Ammonium Perchlorate," Combustion \& Flame, 20, 389-400 (1973)
48 J D Renick, P A Persson and J A Sanchez, "Detonation Properties of Mixtures of HMX and Emulsion Explosives," Proceedings Ninth Symposium (Inter national) on Detonation, Portland, OR, August 28-September 1, 1989, I, 545552

49 H Eyring, R E Powell, G H Duffey and R B Parlin, "The Stability of Detonation," Chem Rev 45, 69-182 (1949), some data taken from A Parisot and P Laffitte, Congr chim ind, Compt rend 18me congr, Nancy, pp y30-936 (1938), also from MacDougall, uncited

50 G V Dimza, "Critical Detonation Diameter for an Explosive containing an Inert Additive," Combustion, Explosives \& Shock Waves (Russian) 12, 216-218 (1976)

51 R Kh Kurbangalina and L I Patronova, "Effect of a Steel Sheath on the Critical Detonation Diameter of Condensed Explosives," Combustion, Explosives \& Shock Waves (Russian) 12, 587-590 (1976) 


\section{Comparing the Data}

We now consider some simple comparisons of the data Figure 1 shows a detonation velocity, $U_{s}$, -inverse radius plot, where the widest possible spread of data has been taken Infinite radius is at the left, moving to the right causes shrinking of the cylinder Ultimately, the lines die out because of failure, ie the cylinder no longer propagates the detonation because too much energy is lost out the sides Large size cylinders lie to the left, tiny ones to the right

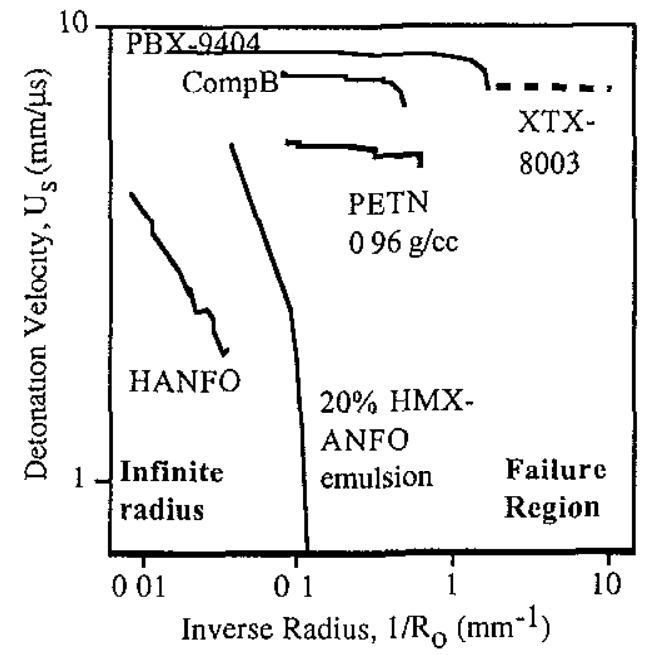

Fig 1 Detonation velocity vs inverse radius for the widest possible spread of explosive data

The most obvious thing about Fig 1 is the huge spread over orders of magnitude It would be nice to reduce to normalized coordinates The most obvious choice for detonation velocity is $U_{\mathrm{S}} / D$ or $1-U_{\mathrm{S}} / D$, where $D$ is the infinite radius detonation velocity It is not possible, however, to select a standard radius because there is no overlap in many cases The only apparent fixed radius is that for failure, $R_{f}$, but this is a function of confinement

From Fig 1, we see varying changes of slope XTX-8003 barely changes its detonation velocity before it fails, whereas the composite explosives can detonate at extremely reduced velocities

The same broad spread appears in Figure 2 when we plot the calculated reaction zone lengths versus cylinder radius The short zones of XTX8003 lie at the lower left and the long zones of HANFO at the upper right

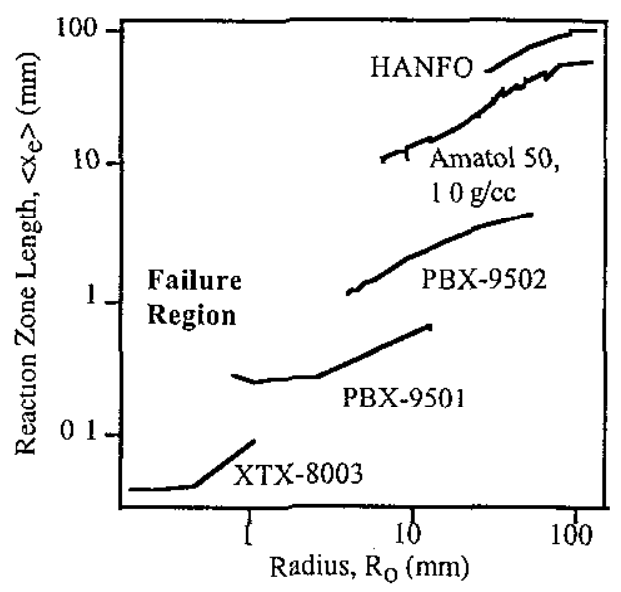

Figure 2 The broad spread of calculated reaction zone lengths The smallest belongs to XTX8003 and the longest to HANFO

Figure 3 shows a family of TNT samples, taken at various densities We see that adding void volume increases both the reaction zone length and the failure radius Also, the reaction 
zone length at a given density increases with radius with a power between 05 and 10

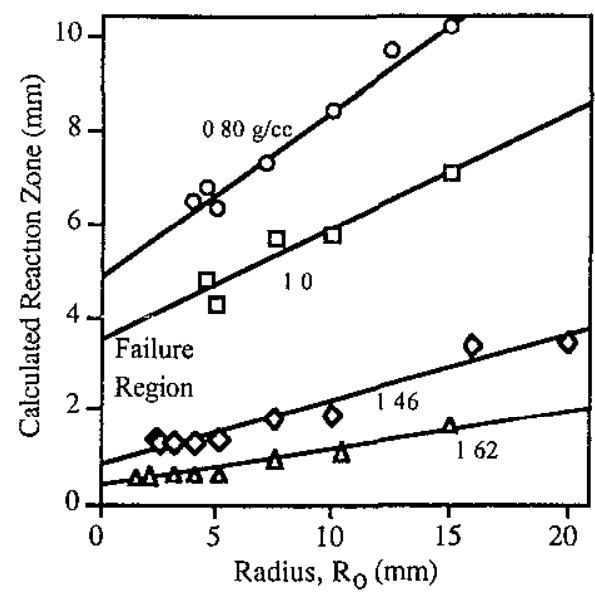

Figure 3 Reaction zone-radius for a family of TNT samples of different densities All work was done by the same researchers The densities are listed

Many other effects appear Figure 4 shows two high-HMX explosives PBX-9501 (95\%) and PBX-9404 (94\%) The two curves overlie each other but the different binders produce different failure radii The binders are PBX9404- NC and CEF and PBX-9501- estane and BDNPA-F

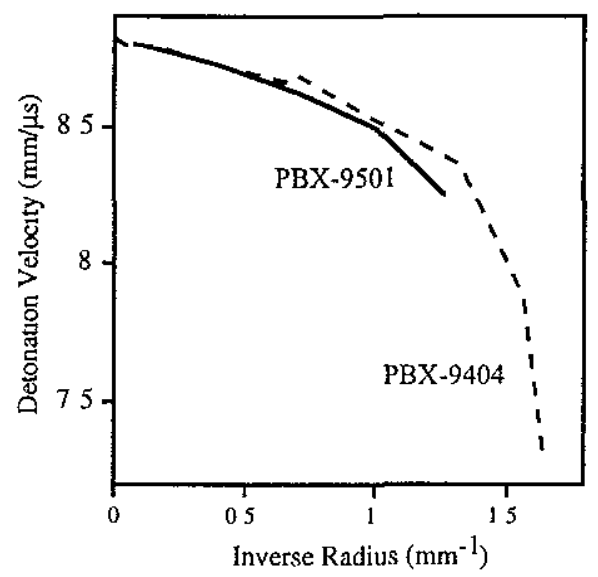

Figure 4 Inverse radius plot for two high-HMX explosives showing that binder affects the failure radius
Figure 5 shows an inverse radius plot for PBXN-111, the only explosive with comparable confined and unconfined data The failure radius is shorter for the unconfined data The reaction zone lengths are about the same The infinite radius detonation velocities are the same

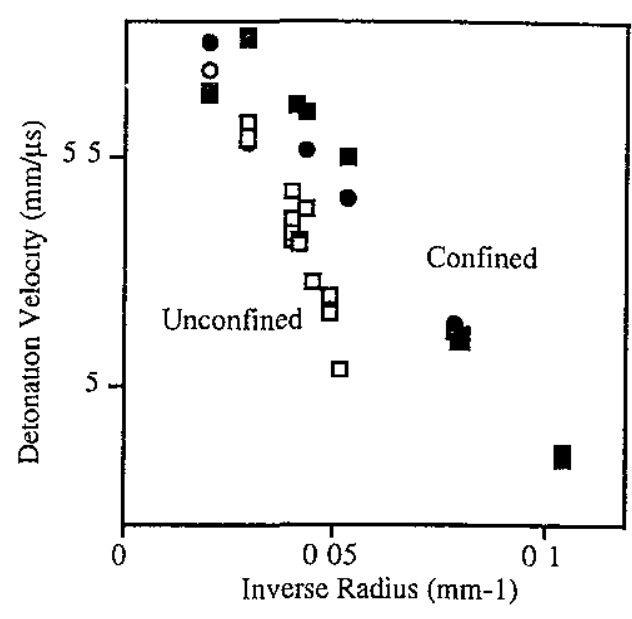

Figure 5 Inverse radius plot of confined (solid symbols) and unconfined (open) PBXN-111 Data is from the U S Navy and Australia Confinement also decreases the failure radius

Figure 6 shows a dramatic reaction zone effect in $70 \% \mathrm{RDX}$-urethane, where particle sizes were carefully set and the explosive was pressed near full density, so there would be almost no hot spots This last feature makes this an unusual explosive in the ranks of those listed in this report The data is also different in that no radius effect is seen The measurements were unfortunately not extended to failure With radius not a factor, the reaction zone lengths are roughly given by

$$
<\mathrm{x}_{\mathrm{c}}>\sim 05+0006 \mathrm{~d}(\mu \mathrm{m})
$$


where $d$ is the RDX particle size NM and liquid TNT show a weak dependence on radius, so that hot spots may be the cause of the radius effect

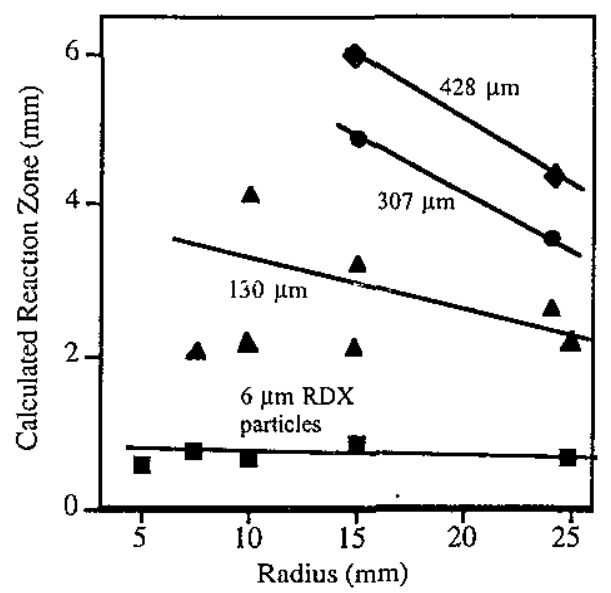

Figure 6 The reaction zone length varies dramatically in these $70 \% \mathrm{RDX}$-urethane samples with well-sieved sizes and almost no hot spots 
Table 1 Detonation front curvature data.

\begin{tabular}{|c|c|c|c|c|c|c|c|c|c|c|c|c|c|c|c|c|c|}
\hline \multirow{4}{*}{ Section } & \multirow{4}{*}{ Explosive } & \multirow{4}{*}{$\begin{array}{c}\text { Den- } \\
\text { sity } \\
\rho_{o} \\
(g / c c)\end{array}$} & \multirow{4}{*}{$\begin{array}{c}\text { Rad- } \\
\text { ius } \\
\mathrm{R}_{0} \\
(\mathrm{~mm})\end{array}$} & \multirow{4}{*}{$\begin{array}{l}\text { Edge } \\
\text { Lag, } \\
\text { Lo }_{0} \\
(\mathrm{~mm})\end{array}$} & \multirow{4}{*}{$\begin{array}{l}\text { calc. } \\
<\mathrm{x}_{\mathrm{e}}> \\
(\mathrm{mm})\end{array}$} & \multicolumn{2}{|c|}{ Det Velocity } & \multirow{2}{*}{\multicolumn{2}{|c|}{$\begin{array}{r}\text { Curvature } \\
\text { fit parameters } \\
\end{array}$}} & \multirow{4}{*}{$\begin{array}{l}\text { Meas. } \\
\text { Edge } \\
\text { Angle } \\
\text { (deg) }\end{array}$} & \multirow{4}{*}{$\begin{array}{l}\text { Calc. } \\
\text { Edge } \\
\text { Angle } \\
\text { (deg) }\end{array}$} & \multirow{4}{*}{$\begin{array}{c}\mathrm{G} \\
\left(\mu \mathrm{s}^{-1}\right. \\
\left.* \mathrm{GPa}^{-2}\right)\end{array}$} & \multirow{4}{*}{$\begin{array}{l}\text { Rcur } \\
1 / 2 \mathrm{~A} \\
(\mathrm{~mm})\end{array}$} & \multirow{3}{*}{\multicolumn{2}{|c|}{ Wall }} & \multirow{4}{*}{$\begin{array}{c}\mathrm{K} \\
(\mathrm{MW} / \\
\left.\mathrm{mm}^{2}\right)\end{array}$} & \multirow{4}{*}{$\begin{array}{l}\text { Len- } \\
\text { gth } \\
(\mathrm{mm})\end{array}$} \\
\hline & & & & & & \multirow{3}{*}{$\begin{array}{c}U_{\mathrm{s}} \\
(\mathrm{mm} / \\
\mu \mathrm{s})\end{array}$} & \multirow{3}{*}{$\begin{array}{c}\mathrm{D} \\
(\mathrm{mm} / \\
\mu \mathrm{s})\end{array}$} & & & & & & & & & & \\
\hline & & & & & & & & $\mathrm{A}_{-1}$ & $\mathrm{~B}_{-5}$ & & & & & & & & \\
\hline & & & & & & & & & $\left(\mathrm{mm}^{-3}\right)$ & & & & & mat & $(\mathrm{mm})$ & & \\
\hline Quad- & $\mathrm{PBX}-9502$ & 1.890 & 6.0 & 0.78 & 14 & 7495 & 778 & $1.28 \mathrm{E}-02$ & $5.69 \mathrm{E}-06$ & 46 & 23 & 0.021 & 39 & & & 85 & \\
\hline ratic & PBX-9502 & 1.890 & 5.0 & 0.74 & 1.2 & 7455 & 778 & $1.68 \mathrm{E}-02$ & $1.91 \mathrm{E}-05$ & 40 & 28 & 0.026 & 30 & & & 74 & \\
\hline ТАТВ & PBX-9502 & 1.890 & 9.0 & 1.03 & 2.0 & 7.553 & 778 & $7.51 \mathrm{E}-03$ & $6.58 \mathrm{E}-07$ & 37 & 20 & 0.016 & 67 & & & 117 & \\
\hline \multirow[t]{12}{*}{ Family } & PBX-9502 & 1.890 & 5.0 & 0.77 & 11 & 7458 & 778 & $1.64 \mathrm{E}-02$ & $2.17 \mathrm{E}-05$ & 40 & 30 & 0.027 & 31 & & & 75 & \\
\hline & $\mathrm{T} 2$ & 1.855 & 25 & 1.92 & 0.9 & 7620 & 765 & $2.25 \mathrm{E}-03$ & $2.08 \mathrm{E}-09$ & 8 & 13 & 0.037 & 222 & & & 46 & \\
\hline & $\mathrm{LX}-17$ & 1.907 & 254 & 2.07 & 2.6 & 7.630 & 772 & $2.42 \mathrm{E}-03$ & $1.91 \mathrm{E}-09$ & 15 & 14 & 0.012 & 207 & $\mathrm{Cu}$ & 2.7 & 136 & 305 \\
\hline & $\mathrm{T} 2$ & 1.855 & 50 & 2.92 & 11 & 7.633 & 765 & $9.23 \mathrm{E}-04$ & $4.38 \mathrm{E}-11$ & 6 & 10 & 0.029 & 542 & & & 56 & \\
\hline & PBX-9502 & 1.890 & 250 & 2.08 & 3.3 & 7.677 & 778 & 2.03E-03 & $2.77 \mathrm{E}-09$ & 42 & 15 & 0.009 & 246 & & & 199 & \\
\hline & PBX-9502 & 1.890 & 25.0 & 2.18 & 34 & 7.672 & 778 & $2.21 \mathrm{E}-03$ & $2.48 \mathrm{E}-09$ & 31 & 14 & 0.009 & 227 & & & 192 & \\
\hline & EDC-35 & 1.904 & 5.0 & & 0.8 & 7440 & 773 & $2.56 \mathrm{E}-02$ & $1.57 \mathrm{E}-05$ & & 29 & 0.036 & 20 & & & 43 & 60 \\
\hline & EDC-35 & 1.904 & 5.0 & & 07 & 7440 & 773 & $2.30 \mathrm{E}-02$ & $3.59 \mathrm{E}-05$ & & 42 & 0.042 & 22 & & & 48 & 60 \\
\hline & EDC-35 & 1.904 & 5.0 & & 0.8 & 7440 & 773 & $2.27 \mathrm{E}-02$ & $2.98 \mathrm{E}-05$ & & 38 & 0.039 & 22 & & & 49 & 60 \\
\hline & EDC-35 & 1.904 & 254 & & 2.0 & 7.670 & 773 & $2.43 E-03$ & $1.24 \mathrm{E}-09$ & & 11 & 0.016 & 206 & & & 98 & 762 \\
\hline & EDC-35 & 1.904 & 254 & & 1.9 & 7.670 & 773 & $2.51 \mathrm{E}-03$ & $1.52 \mathrm{E}-09$ & & 13 & 0.017 & 199 & & & 95 & 762 \\
\hline & EDC-35 & 1.904 & 254 & & 2.0 & 7.670 & 773 & $2.60 \mathrm{E}-03$ & $6.76 \mathrm{E}-10$ & & 10 & 0.016 & 192 & & & 91 & 762 \\
\hline Quad- & $\mathrm{NM}$ & 1118 & 6.35 & 0.21 & 0.2 & 6.199 & 6.21 & $4.36 \mathrm{E}-03$ & $6.66 \mathrm{E}-07$ & 4 & 6 & 0.57 & 115 & $\mathrm{Br}$ & 2.0 & 7 & \\
\hline ratıc & NM & 1118 & 254 & 0.54 & 07 & 6.205 & 6.21 & $6.76 \mathrm{E}-04$ & $5.52 \mathrm{E}-10$ & 1 & 4 & 0.21 & 740 & Py & 2.0 & 21 & \\
\hline $\mathrm{NM}$ & NM & 1124 & 18.42 & 0.85 & 0.3 & 6.230 & 6.24 & $9.50 \mathrm{E}-04$ & $1.16 \mathrm{E}-08$ & 12 & 10 & 040 & 526 & Py & 10 & 18 & \\
\hline \multirow[t]{6}{*}{ Families } & NM & 1124 & 1378 & 0.80 & 0.2 & 6.229 & 6.24 & $1.79 \mathrm{E}-03$ & $6.61 \mathrm{E}-08$ & 16 & 14 & 0.62 & 279 & Py & 10 & 11 & \\
\hline & $\mathrm{NM}$ & I 124 & 9.57 & 0.86 & 04 & 6.208 & 6.24 & $6.32 \mathrm{E}-03$ & $3.52 \mathrm{E}-07$ & 14 & 16 & 0.37 & 79 & $\mathrm{Py}$ & 10 & 13 & \\
\hline & NM-silica & 1171 & 18.59 & 113 & 18 & 6.140 & 6.55 & $9.62 \mathrm{E}-04$ & $174 \mathrm{E}-08$ & 27 & 15 & 0.0077 & 520 & Py & 10 & 1099 & \\
\hline & NM-silica & 1171 & 9.57 & 0.99 & 4.9 & 6.128 & 6.55 & $5.96 \mathrm{E}-03$ & $5.69 \mathrm{E}-07$ & 19 & 21 & 0.028 & 84 & Py & 10 & 182 & \\
\hline & NM-silica & 1.171 & 6.80 & 0.95 & 2.7 & 6.090 & 6.55 & $147 \mathrm{E}-02$ & $2.86 \mathrm{E}-06$ & 21 & 24 & 0.051 & 34 & Py & 10 & 80 & \\
\hline & NM-silica & 1171 & 5.26 & 0.98 & 2.5 & 5.800 & 6.55 & $2.99 \mathrm{E}-02$ & $7.33 \mathrm{E}-06$ & 21 & 26 & 0.051 & 17 & Py & 1.0 & 61 & \\
\hline Quad- & $6 \mu \mathrm{m}$, mono & I 45 & 15 & 0.60 & 11 & 7420 & 745 & $2.51 \mathrm{E}-03$ & $3.15 \mathrm{E}-09$ & 4 & 5 & 0.035 & 199 & & & 42 & 200 \\
\hline ratic & $127 \mu \mathrm{m}, \mathrm{b}_{1} 6$ & 145 & 15 & 140 & 1.8 & 7.330 & 745 & $6.21 \mathrm{E}-03$ & $8.48 \mathrm{E}-09$ & 10 & 13 & 0.021 & 81 & & & 67 & 200 \\
\hline RDX- & $307 \mu \mathrm{m}$, bi 6 & 145 & 15 & 2.00 & 4.5 & 7.030 & 745 & $8.92 \mathrm{E}-03$ & $6.87 \mathrm{E}-09$ & 15 & 17 & 0.008 & 56 & & & 156 & 200 \\
\hline urethane & $428 \mu \mathrm{m}$, mono & 145 & 15 & 5.00 & & 6.870 & 745 & $2.57 \mathrm{E}-02$ & NA & 35 & 38 & & 19 & & & & 200 \\
\hline
\end{tabular}




\begin{tabular}{|c|c|c|c|c|c|c|c|c|c|c|c|c|c|c|c|c|c|c|}
\hline \multirow[b]{2}{*}{ Section } & \multicolumn{2}{|c|}{ Table 1 , page 2} & \multirow[b]{2}{*}{$\mathrm{R}_{\mathrm{o}}$} & \multirow[b]{2}{*}{$\mathrm{L}_{\circ}$} & \multirow[b]{2}{*}{$\mathrm{xe}$} & \multirow[b]{2}{*}{$U_{s}$} & \multirow[b]{2}{*}{$\mathrm{D}$} & \multirow[b]{2}{*}{$\mathrm{A}$} & \multirow[b]{2}{*}{$\mathrm{B}$} & \multirow[b]{2}{*}{ Angle } & \multirow[b]{2}{*}{ Calc. } & \multirow[b]{2}{*}{ G } & \multirow[b]{2}{*}{ Rcur } & \multirow[b]{2}{*}{ mat } & \multirow[b]{2}{*}{$(\mathrm{mm})$} & \multirow[b]{2}{*}{$\mathrm{K}(\mathrm{A})$} & \multirow[b]{2}{*}{$\mathrm{L}$} & \multirow[b]{2}{*}{ ref } \\
\hline & Explosive & $\rho_{0}$ & & & & & & & & & & & & & & & & \\
\hline Misc. & PBX-9404 & 1.840 & 12.7 & 0.46 & 1.9 & 8.750 & 8.80 & $1.01 \mathrm{E}-03$ & $5.68 \mathrm{E}-08$ & 16 & 8 & 0.012 & 495 & & & 254 & & 3 \\
\hline Quadratic & LX-04 & 1.854 & 12.7 & 0.53 & 1.0 & 8.455 & 8.49 & $1.58 \mathrm{E}-03$ & $6.22 \mathrm{E}-08$ & 9 & 9 & 0.025 & 316 & $\mathrm{Cu}$ & 3.2 & 105 & 305 & 1 \\
\hline Homo- and & LX-04 & 1.853 & 12.7 & 0.54 & 2.1 & 8.420 & 8.49 & $2.18 \mathrm{E}-03$ & $3.32 \mathrm{E}-08$ & 14 & 7 & 0.013 & 229 & $\mathrm{Cu}$ & 3.2 & 151 & 305 & 1 \\
\hline Hetero- & LX-04 & 1.863 & 12.7 & 0.58 & 04 & 8.476 & 8.49 & $1.31 \mathrm{E}-03$ & $7.65 \mathrm{E}-08$ & 16 & 10 & 0.063 & 382 & $\mathrm{Cu}$ & 3.2 & 51 & 305 & 1 \\
\hline \multirow[t]{18}{*}{ geneous } & LX-04 & 1.863 & 12.7 & 0.59 & 0.3 & 8.480 & 8.49 & $2.19 \mathrm{E}-03$ & $4.84 \mathrm{E}-08$ & 12 & 9 & 0.099 & 228 & $\mathrm{Cu}$ & 3.2 & 22 & 305 & 1 \\
\hline & PBXN-110 & 1.680 & 24.95 & 2.35 & & 8.390 & & $2.04 \mathrm{E}-03$ & 4.22E-09 & 24 & 19 & & 245 & & & & 178 & 16 \\
\hline & IRX-1 & 1430 & 25 & 2.38 & 4.8 & 7491 & 767 & $2.42 \mathrm{E}-03$ & $374 \mathrm{E}-09$ & 21 & 19 & 0.0080 & 207 & & & 73 & & 17 \\
\hline & PBXW-131 & 1740 & 254 & 0.96 & & 8.644 & & $1.02 \mathrm{E}-03$ & $6.46 \mathrm{E}-10$ & 26 & 5 & & 490 & $\mathrm{Cu}$ & 51 & & 508 & 1 \\
\hline & PBXW-131 & 1740 & 254 & 1.01 & & 8.652 & & $2.07 \mathrm{E}-04$ & $2.57 \mathrm{E}-09$ & 38 & 10 & & 2413 & $\mathrm{Cu}$ & 51 & & 508 & 1 \\
\hline & TNT pressed & 1.621 & 12.7 & 049 & & 6.915 & & $1.82 \mathrm{E}-03$ & 4.12E-08 & 16 & 7 & & 274 & $\mathrm{Cu}$ & 3.2 & & 289 & 1 \\
\hline & Ult. TATB & 1.808 & 12.7 & 1.02 & & 7.503 & & $376 \mathrm{E}-03$ & $7.97 \mathrm{E}-08$ & 18 & 14 & & 133 & $\mathrm{Cu}$ & 3.2 & & 305 & 1 \\
\hline & NQ & 1.66 & 12.7 & 143 & & 8.032 & & $7.24 \mathrm{E}-03$ & $5.91 \mathrm{E}-08$ & 23 & 10 & & 69 & $\mathrm{Cu}$ & 31 & & 298 & \\
\hline & Comp B & 1.670 & 2543 & 174 & 0.3 & 7.860 & 7.87 & $2.40 \mathrm{E}-03$ & $143 \mathrm{E}-09$ & 9 & 12 & 0108 & 208 & & & 19 & & 14 \\
\hline & Comp B & 1.670 & 2543 & 1.85 & 0.3 & 7.860 & 7.87 & $2.48 \mathrm{E}-03$ & $8.00 \mathrm{E}-10$ & 12 & 10 & 0104 & 202 & SS & 12.7 & 18 & & 14 \\
\hline & Octol & 1.809 & 12.7 & 0.61 & 17 & 8.415 & 8.49 & $2.24 \mathrm{E}-03$ & $6.97 \mathrm{E}-08$ & 11 & 11 & 0.015 & 223 & $\mathrm{Cu}$ & 2.6 & 166 & 305 & 1 \\
\hline & RX-08-HD & 1718 & 12.7 & 115 & & 8.147 & & $7.21 \mathrm{E}-03$ & not used & 12 & 10 & & 69 & $\mathrm{Cu}$ & 3.2 & & 305 & 1 \\
\hline & $\mathrm{RX}-52-\mathrm{AD}$ & 1770 & 254 & 169 & & 7.546 & & $1.91 \mathrm{E}-03$ & $1.54 \mathrm{E}-09$ & 13 & 11 & & 262 & $\mathrm{Cu}$ & 2.7 & & 305 & 1 \\
\hline & $\mathrm{RX}-52-\mathrm{AE}$ & 1780 & 254 & 173 & & 7.570 & & $173 \mathrm{E}-03$ & $2.31 \mathrm{E}-09$ & 13 & 13 & & 289 & $\mathrm{Cu}$ & 2.7 & & 305 & 1 \\
\hline & Pentolite & 1.560 & 254 & 570 & & 7190 & & $8.88 \mathrm{E}-03$ & not used & 22 & 24 & & 56 & & & & & 16 \\
\hline & $\mathrm{AP}, 15 \mu \mathrm{m}$ & 0.927 & 26.04 & 414 & & 3.390 & & $6.47 \mathrm{E}-03$ & not used & 17 & 19 & & 77 & $\mathrm{Cu}$ & 40 & & 1067 & 1 \\
\hline & AP, $9 \mu \mathrm{m}$ & 1.020 & 25.0 & 4.72 & 6 & 3600 & 3.80 & $6.65 \mathrm{E}-03$ & $7.25 \mathrm{E}-10$ & 33 & 21 & 0173 & 75 & & & & 229 & 19 \\
\hline & $\mathrm{AP}, 26 \mu \mathrm{m}$ & 1435 & 254 & 5.93 & & 2.400 & & $1.03 \mathrm{E}-02$ & $5.30 \mathrm{E}-09$ & 51 & 41 & & 49 & & & & 114 & 19 \\
\hline \multirow{10}{*}{$\begin{array}{l}\text { Quad- } \\
\text { ratic } \\
\text { PBXN- } \\
111 \\
\text { Families }\end{array}$} & PBXN-11I-N & 1790 & 24.22 & 2.85 & 7 & 5.611 & 5.90 & $4.63 \mathrm{E}-03$ & $9.86 \mathrm{E}-10$ & 16 & 15 & 0.0056 & 108 & $\mathrm{Br}$ & 171 & 59 & 305 & 8,15 \\
\hline & PBXN-111-N & 1790 & 33.96 & 4.00 & 5 & 5757 & 5.90 & $2.90 \mathrm{E}-03$ & $3.52 \mathrm{E}-10$ & 15 & 16 & 0.0077 & 172 & $\mathrm{Br}$ & 7.3 & 48 & 305 & 8 \\
\hline & PBXN-111-A & 179 & 12.60 & 1.60 & 10 & 5.130 & 5.96 & $975 \mathrm{E}-03$ & $1.98 \mathrm{E}-08$ & 19 & 16 & 0.0037 & 51 & $\mathrm{Br}$ & 51 & 73 & 177 & 9 \\
\hline & PBXN-111-A & 179 & 18.94 & 2.10 & 12 & 5406 & 5.96 & $4.86 \mathrm{E}-03$ & $7.81 \mathrm{E}-09$ & 19 & 17 & 0.0033 & 103 & $\mathrm{Br}$ & 50 & 102 & 217 & 9 \\
\hline & PBXN-111-A & 179 & 2349 & 2.50 & 12 & 5.513 & 5.96 & $3.68 \mathrm{E}-03$ & $345 \mathrm{E}-09$ & 20 & 18 & 0.0033 & 136 & $\mathrm{Br}$ & 51 & 111 & 253 & 9 \\
\hline & PBXN-111-A & 179 & 50.00 & & 20 & 5747 & 5.96 & $1.06 \mathrm{E}-03$ & $4.27 \mathrm{E}-11$ & 9 & 11 & 0.0021 & 471 & $\mathrm{Br}$ & 50 & & 378 & 9 \\
\hline & PBXN-111-N & 1790 & 3412 & 574 & 7 & 5.572 & 5.81 & $3.57 \mathrm{E}-03$ & $1.00 \mathrm{E}-09$ & 25 & 28 & 0.0061 & 140 & & & 64 & 305 & 8,14 \\
\hline & PBXN-111-N & 1790 & 24.01 & 4.75 & 8 & 5.309 & 5.81 & $8.21 \mathrm{E}-03$ & $2.66 \mathrm{E}-09$ & 25 & 28 & 0.0051 & 61 & & & 56 & 305 & 8,14 \\
\hline & PBXN-111-N & 1790 & 20.52 & 4.29 & 9 & 5158 & 5.81 & $8.49 \mathrm{E}-03$ & $1.15 \mathrm{E}-08$ & 22 & 31 & 0.0044 & 59 & & & 68 & 305 & 8,14 \\
\hline & PBXN-111-N & 1790 & 24.06 & 4.87 & 8 & 5.311 & 5.81 & $6.46 \mathrm{E}-03$ & $6.16 \mathrm{E}-09$ & 27 & 31 & 0.0047 & 77 & & & 71 & 305 & 8,14 \\
\hline
\end{tabular}


Table 1, Page 3

\begin{tabular}{|c|c|c|c|c|c|c|c|c|c|c|c|c|c|c|c|c|c|c|}
\hline Section & Explosive & $\rho_{\circ}$ & $\mathrm{R}_{0}$ & $L_{o}$ & xe & $U_{s}$ & D & A & B & Angle & Calc. & G & Rcur & mat & $(\mathrm{mm})$ & $\mathrm{K}(\mathrm{A})$ & L & ref \\
\hline & PBXN-111-N & 1790 & 2045 & 4.81 & 8 & 5154 & 5.81 & $9.58 \mathrm{E}-03$ & $1.24 \mathrm{E}-08$ & 29 & 33 & 0.0047 & 52 & & & 61 & 305 & 8,14 \\
\hline & PBXN-111-A & 179 & 49.95 & 6.60 & 17 & 5.688 & 6.04 & $1.58 \mathrm{E}-03$ & $178 \mathrm{E}-10$ & 31 & 26 & 0.0024 & 317 & & & 208 & 378 & 9 \\
\hline & PBXN-111-A & 179 & 34.13 & 5.60 & 14 & 5.527 & 6.04 & $3.38 \mathrm{E}-03$ & $1.13 \mathrm{E}-09$ & 29 & 29 & 0.0029 & 148 & & & 138 & 306 & 9 \\
\hline & PBXN-111-A & 179 & 24.06 & 5.00 & 11 & 5.313 & 6.04 & $6.50 \mathrm{E}-03$ & $6.24 \mathrm{E}-09$ & 33 & 32 & 0.0034 & 77 & & & 98 & 367 & 9 \\
\hline & PBXN-111-A & 179 & 24.01 & 5.30 & 11 & 5.312 & 6.04 & $7.19 \mathrm{E}-03$ & $6.39 \mathrm{E}-09$ & 40 & 33 & 0.0037 & 70 & & & 89 & 329 & 9 \\
\hline & PBXN-111-A & 179 & 20.45 & 4.90 & 10 & 5186 & 6.04 & $9.62 \mathrm{E}-03$ & $1.27 \mathrm{E}-08$ & 32 & 34 & 0.0039 & 52 & & & 76 & 326 & 9 \\
\hline Quad- & ANFO-SW & 116 & 6.8 & \multirow{8}{*}{2.80} & 10 & 3740 & 5.88 & $2.47 \mathrm{E}-02$ & $173 \mathrm{E}-06$ & \multirow{8}{*}{22} & 26 & 0.0061 & 20 & & & 130 & 700 & 10,11 \\
\hline ratic & ANFO-SW & 116 & 8.5 & & 9 & 4.350 & 5.88 & $2.01 \mathrm{E}-02$ & $6.76 \mathrm{E}-07$ & & 28 & 0.0082 & 25 & & & 132 & 700 & 10,11 \\
\hline ANFO & ANFO-SW & 116 & 10.6 & & 9 & 4.530 & 5.88 & $1.58 \mathrm{E}-02$ & $3.53 \mathrm{E}-07$ & & 32 & 0.0081 & 32 & & & 155 & 700 & 10,11 \\
\hline \multirow[t]{12}{*}{ Families } & ANFO-SW & 116 & 10.85 & & 10 & 4.530 & 5.88 & $1.63 \mathrm{E}-02$ & $8.85 \mathrm{E}-08$ & & 23 & 0.0073 & 31 & & & 150 & 700 & 10,11 \\
\hline & ANFO-SW & 116 & 16.8 & & 7 & 5.370 & 5.88 & $8.35 \mathrm{E}-03$ & $1.89 \mathrm{E}-08$ & & 23 & 0.013 & 60 & & & 131 & 700 & 10,11 \\
\hline & ANFO-SW & 116 & 19.0 & & 7 & 5.350 & 5.88 & $8.19 \mathrm{E}-03$ & $9.63 \mathrm{E}-09$ & & 24 & 0.012 & 61 & & & 138 & 520 & 10,11 \\
\hline & ANFO-SW & 116 & 28.3 & & 10 & 5450 & 5.88 & $4.65 \mathrm{E}-03$ & $1.39 \mathrm{E}-09$ & & 23 & 0.0087 & 108 & & & 201 & 600 & 10,11 \\
\hline & ANFO-SW & 116 & 39.3 & & 9 & 5.640 & 5.88 & $2.74 \mathrm{E}-03$ & $4.18 \mathrm{E}-10$ & & 24 & 0.0107 & 182 & & & 197 & 600 & 10,11 \\
\hline & ANFO-NM & 1.248 & 2045 & 4.05 & 20 & 4.870 & 6.46 & $8.19 \mathrm{E}-03$ & $8.28 \mathrm{E}-09$ & 21 & 27 & 0.0040 & 61 & PVC & 37 & 373 & 762 & 12 \\
\hline & ANFO-NM & 1.248 & 38.95 & 715 & 23 & 5.580 & 6.46 & $3.84 \mathrm{E}-03$ & $378 \mathrm{E}-10$ & 20 & 27 & 0.0040 & 130 & PVC & 5.5 & 505 & 915 & 12 \\
\hline & ANFO-NM & 1.248 & 5115 & 8.67 & 23 & 5.840 & 6.46 & $2.66 \mathrm{E}-03$ & $8.77 \mathrm{E}-11$ & 19 & 25 & 0.0042 & 188 & PVC & 6.0 & 537 & 1220 & 12 \\
\hline & HANFO & 1.061 & 60.05 & 13.00 & 65 & 2.900 & 442 & $3.08 \mathrm{E}-03$ & $1.21 \mathrm{E}-10$ & 24 & 43 & 0.0010 & 162 & $\mathrm{Cb}$ & 10 & 711 & 1000 & 9 \\
\hline & HANFO & 1.061 & 76.35 & 17.30 & 68 & 3.280 & 442 & $2.52 \mathrm{E}-03$ & $177 \mathrm{E}-11$ & 26 & 33 & $0001 \mathrm{I}$ & 198 & $\mathrm{Cb}$ & 1.0 & 738 & 1000 & 9 \\
\hline & HANFO & 1.069 & 126.8 & 29.80 & 12 & 4.300 & 442 & $1.58 \mathrm{E}-03$ & $8.88 \mathrm{E}-13$ & 32 & 30 & 0.0079 & 316 & $\mathrm{Cb}$ & 1.0 & 162 & 1000 & 9 \\
\hline & HANFO & 1070 & 40.2 & 12.20 & & 2.386 & 442 & & & & & & & $\mathrm{Cb}$ & 10 & & 1000 & 9 \\
\hline \multirow{6}{*}{$\begin{array}{l}\text { Quad- } \\
\text { ratic } \\
\text { Misc. } \\
\text { Comp- } \\
\text { osite }\end{array}$} & IRX-3A & 1.580 & 25 & 1.87 & 3 & 7787 & 787 & $2.82 \mathrm{E}-03$ & $3.36 \mathrm{E}-10$ & 19 & 9 & 0.024 & 177 & \multirow{5}{*}{$\begin{array}{l}\mathrm{Cu} \\
\mathrm{Cu} \\
\mathrm{Cu}\end{array}$} & \multirow[b]{2}{*}{106} & \multirow{2}{*}{$\begin{array}{c}44 \\
477\end{array}$} & \multicolumn{2}{|r|}{17} \\
\hline & QM100R & 1.509 & 50.8 & 2.91 & 13 & 7429 & 7.59 & $9.91 \mathrm{E}-04$ & $3.11 \mathrm{E}-11$ & 9 & 9 & 0.0045 & 505 & & & & 1016 & 1 \\
\hline & QM100R & 1.509 & 50.8 & 3.29 & 12 & 7420 & 7.59 & $1.26 \mathrm{E}-03$ & $8.23 \mathrm{E}-12$ & 14 & 8 & 0.0048 & 397 & & 106 & 396 & 1016 & 1 \\
\hline & PBXW-126 & 1.800 & 50.8 & 3.00 & & 6.470 & & $8.80 \mathrm{E}-04$ & $4.24 \mathrm{E}-11$ & 8 & 10 & & 568 & & 106 & & 1016 & 1 \\
\hline & IRX-4 & 1.500 & 25 & 376 & 16 & 5.620 & 6.42 & 3.83E-03 & $6.47 \mathrm{E}-09$ & 30 & 30 & 0.0023 & 131 & & & 274 & 178 & 16 \\
\hline & PBXW-123 & 1.920 & 38.5 & 6.55 & & 5.560 & & $3.69 \mathrm{E}-03$ & $6.26 \mathrm{E}-10$ & 21 & 31 & & 136 & $\mathrm{Br}$ & 64 & & 152 & 18 \\
\hline Non- & PETN & 1743 & 12.7 & 010 & & 8.201 & & NA & $2.36 \mathrm{E}-08$ & 3 & 3 & & & $\mathrm{Cu}$ & 3.2 & & 305 & 1 \\
\hline Quadratic & $\mathrm{LX}-14$ & 1.825 & 12.7 & 016 & & 8.778 & & NA & $4.11 \mathrm{E}-08$ & 4 & 5 & & & $\mathrm{Cu}$ & 3.2 & & 305 & 1 \\
\hline & HMX & 1739 & 12.7 & 019 & & 8.550 & & $\mathrm{NA}$ & $372 \mathrm{E}-08$ & 7 & 4 & & & $\mathrm{Cu}$ & & & 305 & 1 \\
\hline & LX-10 & 1.87 & 254 & 0.26 & & 8.820 & & NA & $8.93 \mathrm{E}-10$ & 6 & 3 & & & $\mathrm{Cu}$ & 6.1 & & 305 & 1 \\
\hline & LX-04 & 1.87 & 254 & 0.39 & & 8.470 & & $\mathrm{NA}$ & $1.51 \mathrm{E}-09$ & 7 & 5 & & & $\mathrm{Cu}$ & 6.1 & & 305 & 1 \\
\hline
\end{tabular}


Table I, Page 4

\begin{tabular}{|c|c|c|c|c|c|c|c|c|c|c|c|c|c|c|c|c|c|c|}
\hline Section & Explosive & $\rho_{0}$ & $\mathrm{R}_{0}$ & $\mathrm{~L}_{0}$ & $x_{\mathrm{e}}$ & $\mathrm{U}_{\mathrm{s}}$ & D & A & $\mathrm{B}$ & Angle & Calc. & $\mathrm{G}$ & $\mathrm{R}_{\text {cur }}$ & mat & $(\mathrm{mm})$ & $\mathrm{K}(\mathrm{A})$ & $\mathrm{L}$ & ref \\
\hline Non- & $\mathrm{LX}-19$ & 1.939 & 12.7 & 0.13 & & 9181 & & $\mathrm{NA}$ & NA & 9 & & & & $\mathrm{Cu}$ & 3.2 & & 305 & 1 \\
\hline Quad. & $R X-54-A J$ & 1.82 & 12.8 & 0.55 & & 7.650 & & $N A$ & $1.16 \mathrm{E}-07$ & 13 & & & & $\mathrm{Cu}$ & 2.5 & & 305 & 1 \\
\hline Perturbed & $\mathrm{RX}-08-\mathrm{HD}$ & 1.67 & 12.7 & 0.62 & & 8.035 & & NA & $1.24 \mathrm{E}-07$ & 16 & & & & $\mathrm{Cu}$ & 2.6 & & 305 & 1 \\
\hline \multirow[t]{3}{*}{ Slabs } & EDC-35 & 1.904 & 5.0 & & & 7.56 & 773 & $1.65 \mathrm{E}-02$ & $1.62 \mathrm{E}-05$ & & & & & & & & 60 & 5 \\
\hline & $\mathrm{EDC}-35-40^{\circ} \mathrm{C}$ & 1.904 & 5.0 & & & 7.54 & 773 & $2.09 \mathrm{E}-02$ & $2.31 \mathrm{E}-05$ & & & & & & & & 60 & 5 \\
\hline & EDC-35 & 1.904 & 10.0 & & & 7.62 & 773 & $6.68 \mathrm{E}-03$ & $3.70 \mathrm{E}-07$ & & & & & & & & 120 & 5 \\
\hline
\end{tabular}




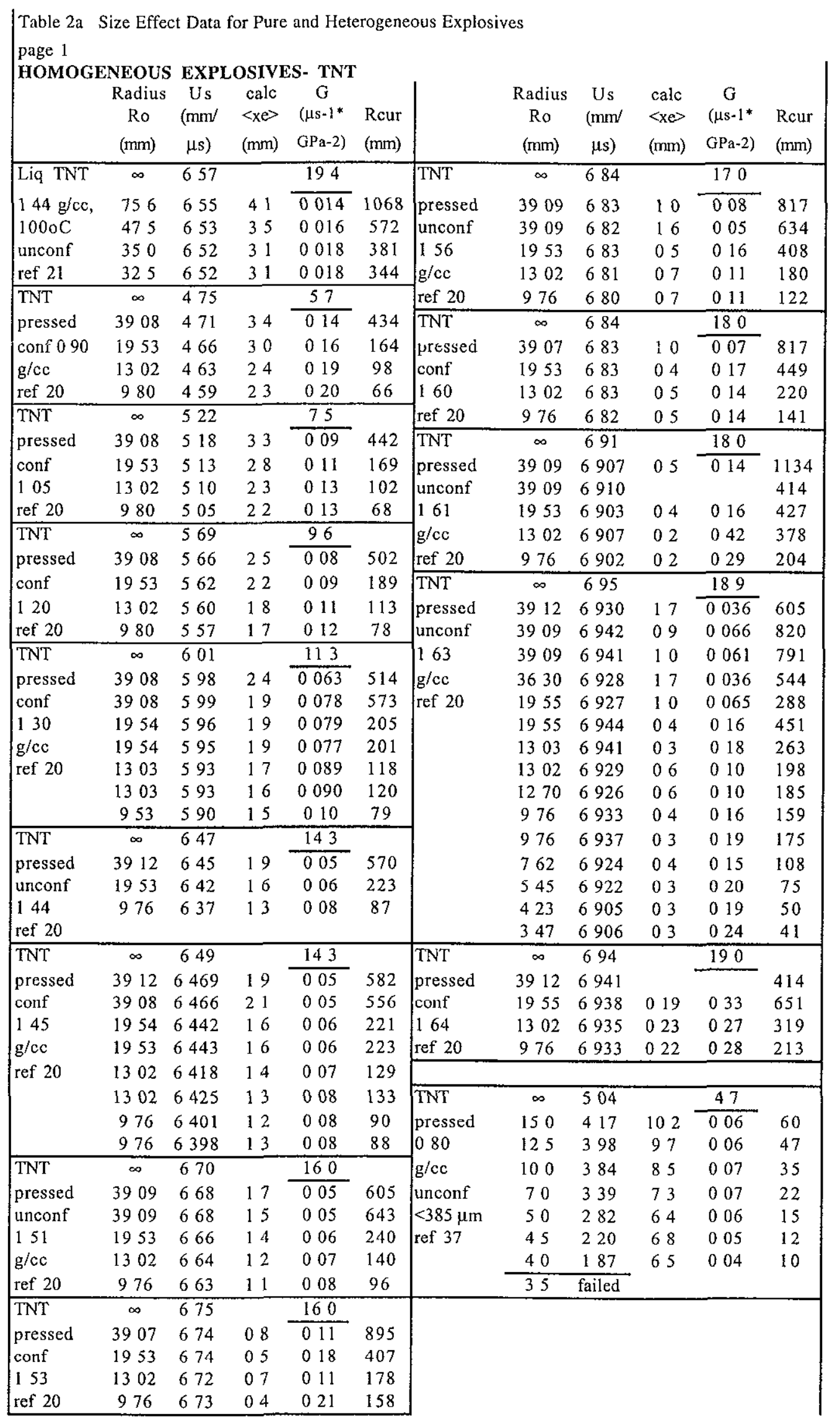




\begin{tabular}{|c|c|c|c|c|c|c|c|c|c|c|c|}
\hline \multirow{2}{*}{$\begin{array}{l}\text { Table } \\
\text { TNT }\end{array}$} & \multirow[b]{2}{*}{ Ro } & \multirow[b]{2}{*}{ Us } & \multirow[b]{2}{*}{$\langle$ xe $\rangle$} & \multirow[b]{2}{*}{$\mathrm{G}$} & \multirow[b]{2}{*}{ Rcur } & \multicolumn{6}{|c|}{ |OTHER HOMOGENEOUS EXPLOSIVES } \\
\hline & & & & & & & Ro & Us & $\langle x e>$ & $\mathrm{G}$ & Rcur \\
\hline TNT & $\infty$ & 523 & & 70 & & TNT & $\infty$ & 709 & & 194 & \\
\hline pressed & 1500 & 471 & 71 & 004 & 71 & cast & 290 & 700 & 35 & 002 & 274 \\
\hline 100 & 1000 & 453 & 58 & 005 & 43 & unconf & 200 & 698 & 28 & 002 & 176 \\
\hline $\mathrm{g} / \mathrm{cc}$ & 750 & 418 & 57 & 005 & 28 & $77 \mathrm{~K}$ & 175 & 700 & 21 & 003 & 165 \\
\hline unconf & 500 & 394 & 43 & 006 & 17 & ref 42 & 160 & 699 & 21 & 003 & 144 \\
\hline$<385 \mu \mathrm{m}$ & 450 & 344 & 48 & 005 & 14 & & 150 & 697 & 21 & 003 & 131 \\
\hline ref 37 & 400 & failed & & & & & 125 & 696 & 19 & 003 & 103 \\
\hline TNT & $\infty$ & 670 & & 145 & & & 100 & 692 & 18 & 003 & 76 \\
\hline pressed & 200 & 655 & 35 & 003 & 156 & & 90 & 688 & 19 & 003 & 64 \\
\hline 146 & 159 & 650 & 34 & 003 & 113 & & 80 & 686 & 18 & 003 & 55 \\
\hline $\mathrm{g} / \mathrm{ec}$ & 100 & 653 & i 9 & 005 & 75 & & 70 & 682 & 17 & 003 & 46 \\
\hline unconf & 745 & 645 & 18 & 005 & 49 & & 60 & 678 & 16 & 004 & 37 \\
\hline$<385 \mu \mathrm{m}$ & 510 & 639 & 14 & 007 & 31 & & 50 & 674 & 15 & 004 & 30 \\
\hline \multirow[t]{5}{*}{ ref 37} & 409 & 633 & 13 & 007 & 24 & & 40 & 675 & 12 & 005 & 24 \\
\hline & 308 & 612 & 13 & 007 & 15 & \multirow{11}{*}{\begin{tabular}{|l} 
TNT \\
cast \\
unconf \\
$20 \mathrm{~K}$ \\
ref 42
\end{tabular}} & $\infty$ & 708 & & 194 & \\
\hline & 258 & 596 & 13 & 007 & 12 & & 280 & 698 & 36 & 002 & 252 \\
\hline & 234 & 577 & 14 & 006 & 10 & & 200 & 699 & 23 & 003 & 192 \\
\hline & 208 & failed & & & & & 160 & 699 & 20 & 003 & 149 \\
\hline TNT & $\infty$ & 693 & & 166 & & & 150 & 695 & 22 & 003 & 126 \\
\hline pressed & 1000 & 685 & 11 & 007 & 97 & & 125 & 697 & 17 & 004 & 111 \\
\hline 155 & 750 & 679 & 12 & 006 & 61 & & 100 & 694 & 16 & 004 & 82 \\
\hline $\mathrm{g} / \mathrm{cc}$ & 500 & 674 & 10 & 008 & 37 & & 90 & 690 & 17 & 003 & 68 \\
\hline unconf & 412 & 672 & 09 & 009 & 29 & & 80 & 689 & 16 & 004 & 60 \\
\hline$<385 \mu \mathrm{m}$ & 258 & 660 & 07 & 010 & 16 & & 70 & 684 & 16 & 004 & 47 \\
\hline \multirow[t]{2}{*}{ ref 37} & 194 & 650 & 07 & 011 & \multirow{2}{*}{$\begin{array}{c}11 \\
8\end{array}$} & & 60 & 676 & 17 & 003 & 37 \\
\hline & 158 & 634 & 07 & 011 & & & & & & & \\
\hline TNT & $\infty$ & 708 & & 205 & & & & & & & \\
\hline pressed & 1500 & 700 & 17 & 003 & 147 & & & & & & \\
\hline 162 & 1035 & 700 & 11 & 005 & 101 & & & & & & \\
\hline $\mathrm{g} / \mathrm{cc}$ & 755 & 698 & 10 & 005 & 69 & & & & & & \\
\hline unconf & 510 & 698 & 07 & 008 & 46 & & & & & & \\
\hline$<385 \mu \mathrm{m}$ & 412 & 694 & 07 & 008 & 34 & & & & & & \\
\hline \multirow[t]{4}{*}{ ref 37} & 310 & 686 & 07 & 008 & 22 & & & & & & \\
\hline & 208 & 675 & 06 & 009 & 13 & & & & & & \\
\hline & 159 & 663 & \multirow[t]{2}{*}{06} & \multirow[t]{2}{*}{009} & \multirow[t]{2}{*}{9} & & & & & & \\
\hline & 103 & failed & & & & & & & & & \\
\hline TNT & $\infty$ & 713 & & 205 & & & & & & & \\
\hline cast & 250 & 695 & 47 & 0011 & 187 & & & & & & \\
\hline 162 & 200 & 695 & 38 & 0014 & 150 & & & & & & \\
\hline $\mathrm{g} / \mathrm{cc}$ & 150 & 692 & 31 & 0017 & 107 & & & & & & \\
\hline unconf & 100 & 682 & 27 & 0019 & 63 & & & & & & \\
\hline \multirow[t]{2}{*}{ ref 37} & 75 & 661 & 29 & 0017 & 40 & & & & & & \\
\hline & 70 & failed & & & & & & & & & \\
\hline TNT & $\infty$ & 696 & & 194 & & & & & & & \\
\hline cast & 280 & 691 & 23 & 003 & 323 & & & & & & \\
\hline unconf & 200 & 690 & 19 & 003 & 208 & & & & & & \\
\hline $291 \mathrm{~K}$ & 175 & 687 & 21 & 003 & 165 & & & & & & \\
\hline 1615 & 150 & 686 & 20 & 003 & 135 & & & & & & \\
\hline $\mathrm{g} / \mathrm{cc}$ & 125 & 685 & 17 & 003 & 110 & & & & & & \\
\hline ref 42 & 100 & 682 & 17 & 003 & 80 & & & & & & \\
\hline & 90 & 680 & 16 & 004 & 70 & & & & & & \\
\hline & 80 & 668 & 21 & 003 & 51 & & & & & & \\
\hline
\end{tabular}




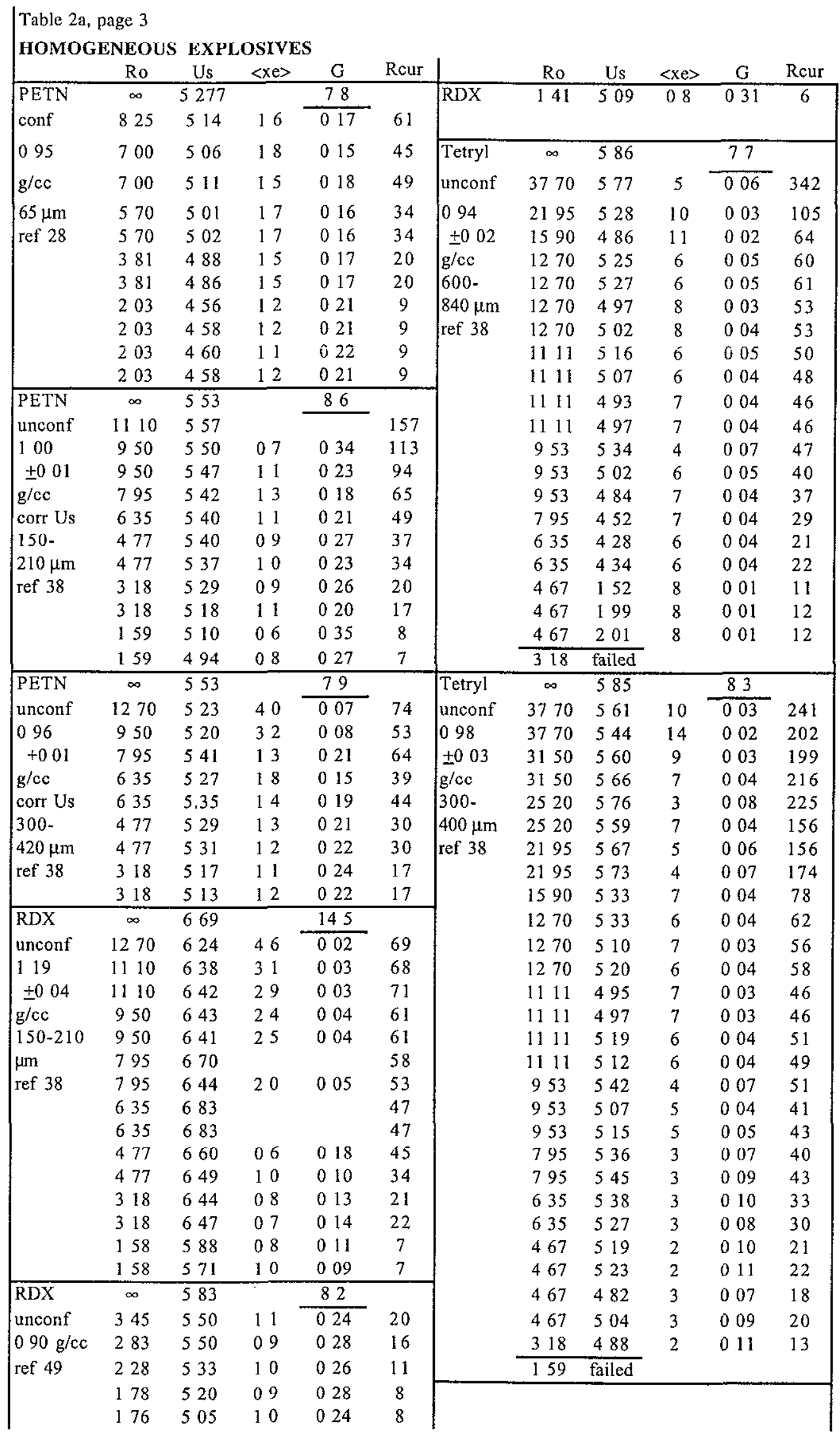




\begin{tabular}{|c|c|c|c|c|c|c|c|c|c|c|c|}
\hline $\begin{array}{l}\text { Table } 2 a \text {, } \\
\text { HOMOG }\end{array}$ & $\begin{array}{l}\text { Dage } 4 \\
\text { ENEOU } \\
\text { Ro }\end{array}$ & $\mathbf{S} \underset{U_{S}}{\mathbf{E X Y}}$ & LOSIV & $\mathrm{VES}_{\mathrm{G}}$ & Rcur & & Ro & & & & \\
\hline ADNBF & $\infty$ & 823 & $2 \mathrm{AC}$ & 253 & & NM & $\frac{\mathrm{n}}{\infty}$ & $\frac{\mathrm{Us}}{6210}$ & $<\mathrm{xe}>$ & $\frac{\mathrm{G}}{119}$ & Rcur \\
\hline 171 & 298 & 781 & 09 & 004 & 18 & conf & 4763 & 6210 & & & \\
\hline $\mathrm{g} / \mathrm{cc}$ & 203 & 772 & 07 & 006 & 11 & 1033 & 1270 & 6201 & 04 & 039 & 247 \\
\hline unconf & 101 & 709 & 06 & 006 & 4 & $\mathrm{~g} / \mathrm{cc}$ & 1269 & 6200 & 04 & 037 & 238 \\
\hline ref 43 & & & & & & $330 \mathrm{C}$ & 6395 & 6188 & 03 & 043 & 92 \\
\hline$\overline{A D N B F}$ & $\infty$ & 826 & & 26.7 & & ref 22 & 6350 & 6190 & 03 & 046 & 94 \\
\hline 175 & 203 & 781 & 06 & 006 & 12 & & 3165 & 6169 & 02 & 057 & 37 \\
\hline $\mathrm{g} / \mathrm{cc}$ & 101 & 731 & 05 & 006 & 5 & & 3215 & 6166 & 03 & 053 & 37 \\
\hline unconf & 101 & 743 & 05 & 007 & 5 & & 1525 & 6125 & 02 & 072 & 14 \\
\hline ref 43 & & & & & & & 1500 & 6128 & 02 & 075 & 14 \\
\hline DNBF & $\infty$ & 755 & & 221 & & & 121 & failed & & & \\
\hline 158 & 638 & 742 & 09 & 005 & 55 & NM & $\infty$ & 6236 & & 119 & \\
\hline $\mathrm{g} / \mathrm{cc}$ & 298 & 741 & 04 & 011 & 25 & unconf & 1842 & 6233 & 02 & 057 & 517 \\
\hline unconf & 203 & 745 & 03 & 019 & 19 & 1130 & 1842 & 6227 & 05 & 027 & 360 \\
\hline ref 43 & 203 & 731 & 04 & 011 & 14 & $\mathrm{~g} / \mathrm{cc}$ & 1546 & 6233 & 02 & 067 & 434 \\
\hline & 101 & 709 & 03 & $\begin{array}{lll}0 & 14 \\
\end{array}$ & 6 & ref 4 & 1378 & 6228 & 04 & 039 & 279 \\
\hline DNBF & $\infty$ & 780 & & 246 & & & 1370 & 6229 & 03 & 043 & 291 \\
\hline 166 & 638 & 762 & 12 & 003 & 49 & & 1106 & 6226 & 03 & 042 & 208 \\
\hline $\mathrm{g} / \mathrm{cc}$ & 298 & 754 & 07 & 006 & 20 & & 967 & 6220 & 04 & 035 & 156 \\
\hline unconf & 298 & 758 & 06 & 007 & 21 & & 958 & 6221 & 04 & 037 & 157 \\
\hline ref 43 & 203 & 746 & 06 & 007 & 13 & & 957 & 6196 & 07 & 019 & 113 \\
\hline & 101 & 679 & 06 & 006 & 4 & & 863 & 6212 & 05 & 030 & 120 \\
\hline DNBF & $\infty$ & 765 & & 255 & & & 833 & 6199 & 06 & 023 & 101 \\
\hline 169 & 203 & 752 & 03 & 013 & 18 & & 832 & 6220 & 03 & 041 & 134 \\
\hline $\mathrm{g} / \mathrm{cc}$ & 101 & 748 & 02 & 021 & 8 & EDNA & $\infty$ & 62 & & 94 & \\
\hline unconf & 076 & 733 & 02 & 018 & 5 & unconf & 9945 & 613 & & & 980 \\
\hline ref 43 & & & & & & 100 & 9945 & 616 & & & 1173 \\
\hline 2,4 & $\infty$ & 433 & & 48 & & \pm 003 & 9945 & 608 & 16 & 0014 & 810 \\
\hline DNT & 1000 & 389 & 5 & 011 & 47 & $\mathrm{~g} / \mathrm{cc}$ & 9945 & 613 & & & 971 \\
\hline unconf & 800 & 378 & 4 & 012 & 36 & $150-$ & 9945 & 570 & & & 512 \\
\hline 095 & 625 & 359 & 4 & 012 & 25 & $210 \mu \mathrm{m}$ & 9945 & 575 & & & 532 \\
\hline $\mathrm{g} / \mathrm{cc}$ & 500 & 327 & 4 & 011 & 17 & ref 38 & 3770 & 638 & & & 277 \\
\hline ref & 313 & 288 & 3 & 012 & 10 & & 3770 & 630 & & & 277 \\
\hline 36 & 250 & 245 & 3 & 011 & 7 & & 1270 & 543 & 7 & 003 & 56 \\
\hline DINGU & $\infty$ & 45 & & 48 & & & 1270 & 552 & 6 & 003 & 58 \\
\hline unconf & 30 & 40 & 15 & 004 & 138 & & 1110 & 532 & 7 & 003 & 47 \\
\hline $080 \mathrm{~g} / \mathrm{cc}$ & 10 & 30 & 11 & 004 & 32 & & 1110 & 538 & 6 & 003 & 48 \\
\hline ref 39 & & & & & & & 950 & 516 & 6 & 003 & 38 \\
\hline DINGU & $\infty$ & 59 & & 82 & & & 950 & 537 & 5 & 004 & 41 \\
\hline $105 \mathrm{~g} / \mathrm{cc}$ & 30 & 52 & 16 & 0016 & 135 & & 795 & 537 & 5 & 004 & 34 \\
\hline ref 39 & 10 & 38 & 11 & 0016 & 31 & & 795 & 545 & 4 & 005 & 35 \\
\hline DINGU & $\infty$ & 646 & & 13 & & & 635 & 534 & 4 & 005 & 27 \\
\hline unconf & 30 & 61 & 10 & 0013 & 174 & & 635 & 547 & 3 & 006 & 29 \\
\hline $130 \mathrm{~g} / \mathrm{cc}$ & 20 & 60 & 8 & 0016 & 107 & & 477 & 499 & 4 & 005 & 18 \\
\hline ref 39 & 10 & 545 & 6 & 0017 & 41 & & 477 & 521 & 3 & 006 & 20 \\
\hline$\overline{\text { DINGU }}$ & $\infty$ & 726 & & 16 & & & 318 & 414 & 3 & 004 & 10 \\
\hline unconf & 20 & 68 & 7 & $\overline{0012}$ & 110 & & 318 & 356 & 4 & 003 & 9 \\
\hline $145 \mathrm{~g} / \mathrm{cc}$ & 10 & 645 & 5 & 0016 & 46 & EDNA & $\infty$ & 61 & & 95 & \\
\hline ref 39 & 6 & 58 & 5 & 0016 & 23 & unconf & 1270 & 549 & 6 & 003 & 60 \\
\hline DINGU & $\infty$ & 75 & & 20 & & 099 & 1270 & 544 & 6 & 003 & 58 \\
\hline unconf & 20 & 725 & 5 & 0012 & 136 & \pm 002 & 1270 & 561 & 5 & 004 & 65 \\
\hline $160 \mathrm{~g} / \mathrm{cc}$ & 10 & 705 & 3 & 0016 & 56 & $\mathrm{~g} / \mathrm{cc}$ & 1270 & 568 & 5 & 004 & 69 \\
\hline ref 39 & 6 & 67 & 3 & 0018 & 28 & 370 & 1110 & 514 & 7 & 003 & 46 \\
\hline & & & & & & $420 \mu \mathrm{m}$ & 1110 & 539 & 6 & 003 & 50 \\
\hline & & & & & & ref 38 & 1110 & 556 & 5 & 004 & 54 \\
\hline & & & & & & & 1110 & 564 & 4 & 005 & 58 \\
\hline
\end{tabular}




\begin{tabular}{|c|c|c|c|c|c|c|c|c|c|c|}
\hline $\begin{array}{l}\text { Table 2a, } \\
\text { HOMOC }\end{array}$ & $\begin{array}{c}\text { page } 5 \\
\text { ENEOUS } \\
\text { Ro }\end{array}$ & $\begin{array}{c}\mathbf{S} \\
\text { EXP } \\
\text { Us }\end{array}$ & $\begin{array}{c}\text { OSIVI } \\
\langle x e\rangle\end{array}$ & & & \multirow[b]{2}{*}{ Ro } & \multirow{2}{*}{ Us } & \multirow{2}{*}{$<x e>$} & \multirow[b]{2}{*}{$\mathrm{G}$} & \multirow{2}{*}{ Rcur } \\
\hline EDNA & $\frac{\text { Ro }}{950}$ & $\frac{\text { Us }}{539}$ & $\frac{\langle x \mathrm{e}\rangle}{5}$ & $\frac{\mathrm{G}}{004}$ & $\frac{\text { Rcur }}{43}$ & & & & & \\
\hline unconf & 950 & $\begin{array}{l}539 \\
532\end{array}$ & 5 & 004 & 42 & & & & & \\
\hline 099 & 950 & 556 & 4 & 005 & 46 & & & & & \\
\hline $\mathrm{g} / \mathrm{cc}$ & 950 & 558 & 4 & 005 & 47 & & & & & \\
\hline unconf & 795 & 500 & 6 & 003 & 31 & & & & & \\
\hline 370 & 795 & 522 & 5 & 004 & 34 & & & & & \\
\hline $420 \mu \mathrm{m}$ & 795 & 547 & 4 & 005 & 37 & & & & & \\
\hline ref 38 & 795 & 534 & 4 & 004 & 35 & & & & & \\
\hline & 635 & 521 & 4 & 005 & 27 & & & & & \\
\hline & 635 & 477 & 5 & 003 & 24 & & & & & \\
\hline & 635 & 520 & 4 & 005 & 26 & & & & & \\
\hline & 635 & 524 & 4 & 005 & 26 & & & & & \\
\hline & 477 & 409 & 5 & 003 & 15 & & & & & \\
\hline & 477 & 486 & 4 & 005 & 18 & & & & & \\
\hline & 477 & 486 & 4 & 005 & 18 & & & & & \\
\hline & 477 & 498 & 3 & 005 & 19 & & & & & \\
\hline & 318 & 346 & 4 & 003 & 9 & & & & & \\
\hline & 318 & 349 & 4 & 003 & 9 & & & & & \\
\hline NTO & $\infty$ & 778 & & 25 & & & & & & \\
\hline 175 & 20 & 772 & 17 & $\begin{array}{lll}0 & 02\end{array}$ & 222 & & & & & \\
\hline $\mathrm{g} / \mathrm{cc}$ & 14 & 774 & 09 & 004 & 177 & & & & & \\
\hline unconf & 10 & 765 & 15 & 003 & 85 & & & & & \\
\hline $450 \mu \mathrm{m}$ & 9 & 769 & 10 & 004 & 87 & & & & & \\
\hline ref 25 & 7 & failed & & & & & & & & \\
\hline NTO & $\infty$ & 842 & & 286 & & & & & & \\
\hline 184 & 14 & 819 & 3 & 0011 & 102 & & & & & \\
\hline $\mathrm{g} / \mathrm{cc}$ & 10 & 804 & 3 & 0011 & 62 & & & & & \\
\hline unconf & 9 & 797 & 3 & 0011 & 52 & & & & & \\
\hline $\operatorname{ref} 25$ & 7 & 793 & 2 & 0013 & 40 & & & & & \\
\hline NTO & $\infty$ & 823 & & 286 & & & & & & \\
\hline 185 & 20 & 809 & 3 & 0011 & 172 & & & & & \\
\hline$g / c c$ & 14 & 812 & 2 & 0018 & 129 & & & & & \\
\hline unconf & 10 & 802 & 2 & 0017 & 75 & & & & & \\
\hline $450 \mu \mathrm{m}$ & 9 & 797 & 2 & 0016 & 63 & & & & & \\
\hline ref 25 & 8 & failed & & & & & & & & \\
\hline NTO & $\infty$ & 825 & & 286 & & & & & & \\
\hline 187 & 1800 & 818 & 2 & 0019 & 192 & & & & & \\
\hline $\mathrm{g} / \mathrm{cc}$ & 1626 & 816 & 2 & 0019 & 163 & & & & & \\
\hline unconf & 1446 & 817 & 1 & 0022 & 150 & & & & & \\
\hline $250 \mu \mathrm{m}$ & 12.88 & 814 & 2 & 0021 & 121 & & & & & \\
\hline LANL & 1268 & 814 & 2 & 0021 & 121 & & & & & \\
\hline ref 24 & 1270 & failed & & & & & & & & \\
\hline picric & $\infty$ & 525 & & 62 & & & & & & \\
\hline acid & 857 & 492 & 3 & 014 & 47 & & & & & \\
\hline $090 \mathrm{~g} / \mathrm{cc}$ & 435 & 458 & 2 & 016 & 19 & & & & & \\
\hline unconf & 390 & 442 & 3 & 015 & 16 & & & & & \\
\hline ref 49 & 306 & 432 & 2 & 017 & 12 & & & & & \\
\hline & 248 & 406 & 2 & 017 & 9 & & & & & \\
\hline & 208 & 408 & 2 & 020 & 7 & & & & & \\
\hline & 203 & 369 & 2 & 016 & 7 & & & & & \\
\hline & 152 & 248 & 2 & 010 & 4 & & & & & \\
\hline & 136 & 223 & 2 & 009 & 4 & & & & & \\
\hline
\end{tabular}




\begin{tabular}{|c|c|c|c|c|c|c|c|c|c|c|c|}
\hline HETER & $\begin{array}{l}\text { page } 6 \\
\text { DGENE }\end{array}$ & OUS $-<2$ & $\%$ BII & DER & & & & & & & \\
\hline & Ro & Us & $<x e>$ & $\mathrm{G}$ & Rcur & & Ro & Us & $\langle x e\rangle$ & $\mathrm{G}$ & Rcur \\
\hline XIX- & $\infty$ & 7307 & & 18 & & $\mathrm{NM}$ & 477 & 4916 & 4 & 003 & 18 \\
\hline 8003 & 102 & 7248 & 009 & 08 & 112 & silica & last one & failing & & & \\
\hline 153 & 045 & 7244 & 004 & 17 & 48 & $\mathrm{X}-0219$ & $\infty$ & 7684 & & 263 & \\
\hline $\mathrm{g} / \mathrm{cc}$ & 026 & 7167 & 004 & 17 & 21 & unconf & 2540 & 7555 & 4 & 0010 & 220 \\
\hline unconf & 019 & 7087 & 004 & 17 & 14 & 191 & 2060 & 7531 & 3 & 0010 & 168 \\
\hline ref 22 & & & & & & $g / c c$ & 1270 & 7462 & 3 & 0013 & 92 \\
\hline Comp & $\infty$ & 8300 & & 27 & & ref 22 & 1270 & 7457 & 3 & 0013 & 90 \\
\hline A & 1269 & 8262 & 08 & 005 & 167 & & 1270 & 7453 & 3 & 0013 & 90 \\
\hline 169 & 635 & 8254 & 04 & 008 & 79 & & 900 & 7397 & 2 & 0015 & 60 \\
\hline $\mathrm{g} / \mathrm{cc}$ & 423 & 8236 & 04 & 010 & 47 & & 795 & 7380 & 2 & 0017 & 52 \\
\hline ref 22 & 318 & 8213 & 03 & 011 & 32 & & 700 & failed & & & \\
\hline & 254 & 8172 & 03 & 010 & 22 & EDC-35 & $\infty$ & 772 & & 275 & \\
\hline & 212 & 8143 & 03 & 011 & 18 & 190 & 6350 & 7690 & 35 & 0009 & 886 \\
\hline $\mathrm{PBX}-$ & $\infty$ & 8805 & & 345 & & $\mathrm{~g} / \mathrm{cc}$ & 2540 & 767 & 27 & 0012 & 300 \\
\hline 9404 & 7300 & 8800 & 11 & 002 & 1941 & ref 5 , & 2540 & 7639 & 27 & 0012 & 255 \\
\hline unconf & 7300 & 8803 & 06 & 004 & 2631 & 29 & 1270 & 7586 & 19 & 0017 & 108 \\
\hline 183 & 1905 & 8789 & 06 & 004 & 342 & & 635 & 7485 & 14 & 0023 & 45 \\
\hline $\mathrm{g} / \mathrm{cc}$ & 1270 & 8774 & 06 & 004 & 184 & & 500 & 744 & 12 & 003 & 33 \\
\hline ref 22 & 1270 & 8775 & 06 & 004 & 185 & $\overline{E D C-35}$ & $\infty$ & 792 & & 275 & \\
\hline & 1145 & 8793 & 03 & 008 & 226 & $-40 \mathrm{oC}$ & 6350 & 7813 & 8 & 0004 & 585 \\
\hline & 816 & 8789 & 03 & 009 & 147 & ref 29 & 2540 & 7648 & 6 & 0005 & 172 \\
\hline & 635 & 8776 & 03 & 008 & 94 & & 1270 & 7586 & 3 & 0009 & 80 \\
\hline & 319 & 8731 & 03 & 008 & 34 & & 635 & 7423 & 2 & 0014 & 35 \\
\hline & 144 & 8651 & 02 & 011 & 12 & PBX- & $\infty$ & 7.78 & & 275 & \\
\hline & 140 & 8668 & 02 & 012 & 12 & 9502 & 5400 & 7729 & 42 & 0008 & 637 \\
\hline & 100 & 8525 & 02 & 010 & 7 & unconf & 2500 & 7664 & 33 & 0010 & 221 \\
\hline & 075 & 8355 & 02 & 010 & 4 & 1890 & 2500 & 7667 & 33 & 0010 & 225 \\
\hline & 064 & 7874 & 03 & 007 & 3 & \pm 0003 & 1575 & 7624 & 26 & 0013 & 128 \\
\hline & 061 & 7279 & 04 & 005 & 2 & $\mathrm{~g} / \mathrm{cc}$ & 1165 & 7586 & 22 & 0015 & 87 \\
\hline & 058 & failed & & & & $24^{\circ} \mathrm{C}$ & 900 & 7557 & 19 & 0017 & 65 \\
\hline PBX- & $\infty$ & 882 & & 351 & & ref 26 & 900 & 7552 & 19 & 0017 & 64 \\
\hline 9501 & 1270 & 879 & 06 & 004 & 185 & & 600 & 7503 & 14 & 0022 & 40 \\
\hline unconf & 1270 & 8791 & 06 & 004 & 187 & & 500 & 7475 & 13 & 0025 & 33 \\
\hline 183 & 1270 & 8792 & 06 & 004 & 191 & & 450 & 7456 & 12 & 003 & 28 \\
\hline $\mathrm{g} / \mathrm{cc}$ & 251 & 8728 & 03 & 009 & 25 & & 425 & 742 & 12 & 003 & 26 \\
\hline ref 22 & 142 & 8612 & 03 & 009 & 11 & & 400 & 7421 & 11 & 003 & 24 \\
\hline & 101 & 8487 & 02 & 009 & 7 & & 375 & failed & & & \\
\hline & 079 & 8259 & 03 & 008 & 4 & PBX- & $\infty$ & 7723 & & 275 & \\
\hline NM- & $\infty$ & 6550 & & 11 & & 9502 & 2500 & 7648 & 25 & 0013 & 257 \\
\hline silica- & 1860 & 6143 & 6 & 003 & 104 & $750 \mathrm{C}$ & 1650 & 7614 & 21 & 0015 & 150 \\
\hline guar & 1845 & 614 & 25 & 006 & 162 & unconf & 900 & 7548 & 16 & 0020 & 70 \\
\hline unconf & 957 & 6128 & 14 & 012 & 82 & 1892 & 600 & 7501 & 12 & 0026 & 43 \\
\hline 117 & 895 & 6113 & 14 & 011 & 73 & $\pm 0 \quad 002$ & 500 & 7477 & 11 & 003 & 34 \\
\hline $\mathrm{g} / \mathrm{cc}$ & 680 & 6086 & 12 & 013 & 52 & ref 26 & 400 & 7439 & 10 & 003 & 26 \\
\hline ref 4 & 674 & 6093 & 12 & 013 & 52 & & 300 & 7377 & 08 & 004 & 19 \\
\hline & 663 & 6071 & 13 & 012 & 49 & & failed & & & & \\
\hline & 581 & 5978 & 15 & 010 & 37 & PBX- & $\infty$ & 7691 & & 275 & \\
\hline & 578 & 5973 & 15 & 010 & 37 & 9502 & 2500 & 767 & 11 & 0030 & 392 \\
\hline & 550 & 5777 & 21 & 007 & 29 & $-55 \mathrm{oC}$ & 2500 & 7665 & 12 & 0026 & 367 \\
\hline & 543 & 5818 & 20 & 008 & 29 & unconf & 1650 & 7616 & 17 & 0019 & 170 \\
\hline & 543 & 5893 & 17 & 009 & 31 & 1891 & 900 & 7546 & 14 & 0023 & 75 \\
\hline & 543 & 5854 & 19 & 008 & 30 & $\pm 0 \quad 002$ & 715 & 7485 & 14 & 0022 & 53 \\
\hline & 531 & 5872 & 18 & 009 & 30 & ref 26 & 600 & 7465 & 13 & 0025 & 43 \\
\hline & 527 & 5772 & 21 & 007 & 28 & & 550 & 7424 & 13 & 0024 & 37 \\
\hline & 526 & 5869 & 18 & 009 & 30 & & 525 & 7398 & 13 & 0024 & 34 \\
\hline & 507 & 5399 & 29 & 005 & 22 & & & & & & \\
\hline
\end{tabular}




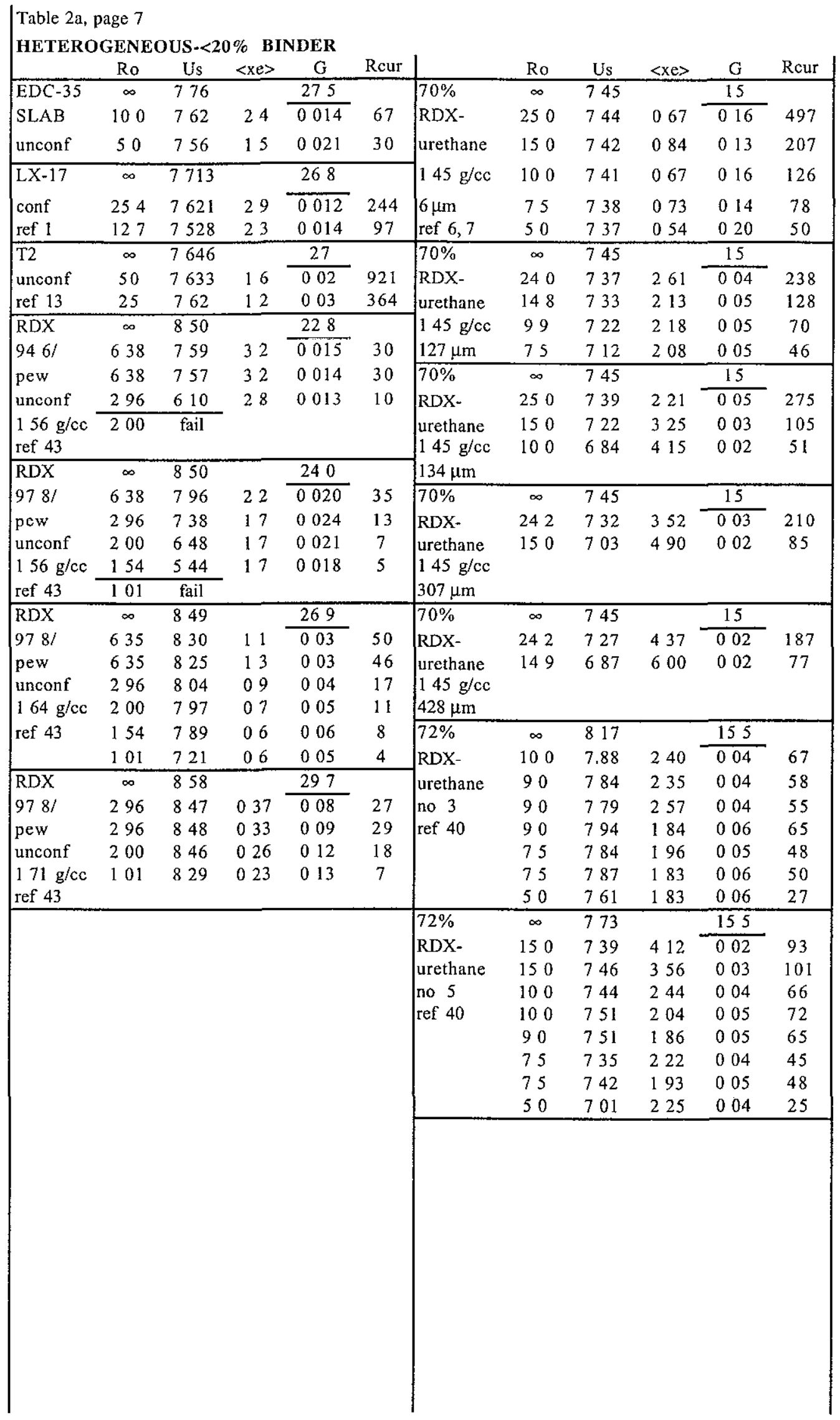


Table 2b Size Effect Data for Binary and Composite Explosives BINARY EXLOSIVES

\begin{tabular}{|c|c|c|c|c|c|c|c|c|c|c|c|}
\hline & Ro & Us & $\langle x e\rangle$ & $G$ & Rcur & & Ro & Us & $\langle$ xe $\rangle$ & $\mathrm{G}$ & Rcur \\
\hline Comp B & $\infty$ & 792 & & 268 & & Octol & $\infty$ & 8472 & & 322 & \\
\hline Type I & 1275 & 7868 & 10 & 004 & 149 & unconf & 2540 & 8452 & 10 & 003 & 418 \\
\hline$<400 \mu \mathrm{m}$ & 1275 & 7887 & 07 & 005 & 175 & 182 & 1906 & 845 & 08 & 003 & 305 \\
\hline $\mathrm{RDX}$ & 1240 & 7869 & 09 & 004 & 146 & $\mathrm{~g} / \mathrm{cc}$ & 1145 & 8415 & 09 & 003 & 134 \\
\hline 171 & 1240 & 7864 & 10 & 003 & 143 & ref 22 & 1145 & 8427 & 08 & 003 & 144 \\
\hline $\mathrm{g} / \mathrm{cc}$ & 1240 & 7847 & 12 & 003 & 130 & & 815 & 8402 & 07 & 004 & 89 \\
\hline \multirow[t]{22}{*}{ ref 23} & 635 & 7816 & 08 & 004 & 59 & & 815 & 84 & 07 & 003 & 88 \\
\hline & 635 & 7819 & 08 & 005 & 59 & & 636 & 8357 & 08 & 003 & 59 \\
\hline & 500 & 7787 & 07 & 005 & 43 & & 317 & 8161 & 08 & 003 & 21 \\
\hline & 500 & 7792 & 07 & 005 & 44 & $\mathrm{X}-0341$ & $\infty$ & 782 & & 281 & \\
\hline & 500 & 7755 & 08 & 004 & 40 & $190 \mathrm{~g} / \mathrm{cc}$ & 250 & 775 & 24 & 001 & 265 \\
\hline & 424 & 7738 & 08 & 005 & 33 & unconf & 90 & 764 & 16 & 0019 & 69 \\
\hline & 424 & 7742 & 07 & 005 & 33 & ref 41 & 45 & 748 & 12 & 0025 & 28 \\
\hline & 398 & 7738 & 07 & 005 & 31 & HMX & 40 & 741 & 12 & 0024 & 23 \\
\hline & 398 & 7725 & 07 & 005 & 30 & $15 \mu \mathrm{m}$ & & & & & \\
\hline & 398 & 7746 & 07 & 005 & 32 & TATB & & & & & \\
\hline & 318 & 7648 & 07 & 005 & 22 & $60 \mu \mathrm{m}$ & & & & & \\
\hline & 318 & 7650 & 07 & 005 & 22 & $\mathrm{X}-0342$ & $\infty$ & 787 & & 285 & \\
\hline & 2.81 & 7572 & 08 & 004 & 18 & $190 \mathrm{~g} / \mathrm{cc}$ & 250 & 782 & 19 & 002 & 296 \\
\hline & 281 & 7561 & 08 & 004 & 18 & unconf & 90 & 772 & 14 & 002 & 73 \\
\hline & 255 & 7476 & 08 & 004 & 15 & ref 41 & 45 & 759 & 11 & 003 & 30 \\
\hline & 254 & 7476 & 08 & 004 & 14 & & 40 & 756 & 10 & 003 & 26 \\
\hline & 232 & 7326 & 09 & 004 & 12 & & 35 & 750 & 10 & 003 & 21 \\
\hline & 230 & 7308 & 09 & 004 & 12 & $X-0343$ & $\infty$ & 791 & & 290 & \\
\hline & 223 & 7092 & 11 & 003 & 10 & $190 \mathrm{~g} / \mathrm{cc}$ & 250 & 788 & 13 & 002 & 360 \\
\hline & 222 & 7066 & 11 & 003 & 10 & unconf & 90 & 779 & 12 & 002 & 81 \\
\hline & 214 & 6709 & 13 & 002 & 9 & ref 41 & 45 & 770 & 09 & 003 & 33 \\
\hline & 214 & failed & & & & & 35 & 764 & 08 & 004 & 23 \\
\hline Comp B & $\infty$ & 792 & & 268 & & & 30 & 759 & 08 & 004 & 19 \\
\hline Type II & 1245 & 7743 & 22 & 002 & 97 & $\mathrm{X}-0344$ & $\infty$ & 805 & & 295 & \\
\hline $400-800$ & 1242 & 7789 & 18 & 002 & 107 & $189 \mathrm{~g} / \mathrm{cc}$ & 250 & 803 & 11 & 003 & 389 \\
\hline$\mu \mathrm{m}$ & 1245 & 7787 & 18 & 002 & 108 & unconf & 90 & 796 & 10 & 003 & 89 \\
\hline RDX & 637 & 7631 & 15 & 002 & 42 & ref 41 & 45 & 787 & 08 & 004 & 35 \\
\hline 171 & 637 & 7643 & 15 & 002 & 43 & HMX & 40 & 785 & 08 & 004 & 30 \\
\hline $\mathrm{g} / \mathrm{cc}$ & 637 & 7664 & 14 & 002 & 44 & $15 \mu \mathrm{m}$ & 35 & 783 & 07 & 004 & 26 \\
\hline \multirow[t]{11}{*}{ ref 23} & 424 & 7503 & 13 & 003 & 25 & TATB & 30 & 780 & 06 & 004 & 21 \\
\hline & 424 & 7516 & 13 & 003 & 25 & $60 \mu \mathrm{m}$ & 20 & 767 & 06 & 005 & 12 \\
\hline & 420 & 7485 & 13 & 002 & 24 & PBXW-7 & $\infty$ & 756 & & 173 & \\
\hline & 391 & 7394 & 14 & 002 & 21 & Type II & 638 & 732 & 14 & 006 & 44 \\
\hline & 391 & 7481 & 12 & 003 & 23 & $151 \mathrm{~g} / \mathrm{cc}$ & 296 & 679 & 14 & 005 & 14 \\
\hline & 375 & 7372 & 14 & 002 & 20 & ref 43 & 154 & 633 & 10 & 007 & 6 \\
\hline & 375 & 7458 & 12 & 003 & 21 & PBXW-7 & $\infty$ & 792 & & 230 & \\
\hline & 358 & 7233 & 15 & 002 & 18 & Type II & 638 & 778 & 09 & 005 & 54 \\
\hline & 360 & 7268 & 15 & 002 & 19 & $170 \mathrm{~g} / \mathrm{cc}$ & 638 & 769 & 13 & 004 & 46 \\
\hline & 356 & 7369 & 13 & 002 & 19 & unconf & 638 & 789 & 03 & 015 & 94 \\
\hline & 356 & 7273 & 15 & 002 & 18 & ref 43 & 296 & 749 & 09 & 005 & 17 \\
\hline Cyclotol & $\infty$ & 8208 & & 294 & & & 296 & 740 & 11 & 004 & 16 \\
\hline unconf & 5080 & 8217 & & & & & 296 & 760 & 08 & 006 & 19 \\
\hline 174 & 2540 & 8204 & & & & & 201 & 740 & 07 & 006 & 11 \\
\hline $\mathrm{g} / \mathrm{cc}$ & 1270 & 8160 & 09 & 003 & 155 & & 201 & 744 & 07 & 007 & 11 \\
\hline ref 22 & 845 & 8107 & 10 & 003 & 80 & & 154 & 728 & 06 & 007 & 8 \\
\hline probably & 635 & 8116 & 07 & 004 & 63 & & 101 & fail & & & \\
\hline \multirow[t]{4}{*}{$77-23$} & 425 & 8012 & 08 & 004 & 32 & BX-4 & $\infty$ & 790 & & 248 & \\
\hline & 365 & 7859 & 10 & 003 & 23 & $175 \mathrm{~g} / \mathrm{cc}$ & 1270 & 780 & 16 & 0026 & 118 \\
\hline & 320 & 7664 & 12 & 002 & 17 & unconf & 1000 & 778 & 13 & 0030 & 89 \\
\hline & 280 & failed & & & & ref 43 & 635 & 770 & 12 & 0033 & 47 \\
\hline
\end{tabular}




\begin{tabular}{|c|c|c|c|c|c|c|c|c|c|c|c|}
\hline \multicolumn{12}{|c|}{$\begin{array}{l}\text { Table } 2 \mathrm{~b} \text {, page } 2 \\
\text { COMPOSITES - ANFO }\end{array}$} \\
\hline & Ro & Us & $\langle x e\rangle$ & $\mathrm{G}$ & Rcur & & Ro & Us & $\langle\mathrm{xe}\rangle$ & $\mathrm{G}$ & Reur \\
\hline \multirow{8}{*}{$\begin{array}{l}\text { QM-100 } \\
\text { unconf } \\
126 \\
\mathrm{~g} / \mathrm{cc} \\
\text { ref } 1 \text {, } \\
35\end{array}$} & $\infty$ & 653 & \multicolumn{3}{|c|}{10} & \multirow{5}{*}{$\begin{array}{l}\text { ANFO } \\
080 \mathrm{~g} / \mathrm{cc} \\
\text { unconf, } \\
\text { ref } 32\end{array}$} & \multicolumn{2}{|r|}{474} & \multicolumn{3}{|c|}{46} \\
\hline & 15240 & 629 & 37 & 0006 & 1008 & & 1460 & 456 & 36 & 0019 & 944 \\
\hline & 7620 & 605 & 29 & 0007 & 407 & & 1460 & 455 & 38 & 0018 & 933 \\
\hline & 5080 & 581 & 26 & 0008 & 233 & & 510 & 389 & 36 & 0016 & \multirow{2}{*}{$\begin{array}{c}200 \\
81\end{array}$} \\
\hline & 3810 & 558 & 23 & 0008 & 159 & & 255 & 325 & 26 & 0019 & \\
\hline & 3048 & 538 & 21 & 0009 & 120 & \multirow{2}{*}{$\begin{array}{l}\text { HANFO } \\
1069\end{array}$} & $\infty$ & 5350 & \multicolumn{3}{|c|}{62} \\
\hline & 2540 & 510 & 20 & 0009 & 92 & & $\begin{array}{c}1268 \\
5\end{array}$ & 4302 & 94 & 0004 & 487 \\
\hline & 1905 & 462 & 18 & 0009 & 62 & $g / c c$ & 9240 & 3732 & 92 & 0003 & 302 \\
\hline ANFO & $\infty$ & 588 & & 92 & & ref 9 & 9240 & 3512 & 100 & 0003 & 285 \\
\hline & 4400 & 562 & 12 & 0017 & 272 & & 7635 & 3277 & 89 & 0003 & 228 \\
\hline unconf & 4400 & 561 & 12 & 0017 & 269 & & 6005 & 2904 & 78 & 0003 & 174 \\
\hline 116 & 3930 & 564 & 10 & 0021 & 251 & & 5040 & 2581 & 71 & 0003 & 139 \\
\hline $\mathrm{g} / \mathrm{cc}$ & 2830 & 545 & 11 & 0019 & 151 & & 5040 & 2657 & 70 & 0003 & 141 \\
\hline ref 10 & 2400 & 538 & 10 & 0020 & 120 & & 4485 & 2364 & 67 & 0003 & 123 \\
\hline 11 & 1900 & 535 & 8 & 0024 & 93 & & 4015 & 2386 & 60 & 0003 & 110 \\
\hline & 1700 & 521 & 9 & 0022 & 76 & & 3510 & 2288 & 53 & 0004 & 91 \\
\hline & 1700 & 507 & 10 & 0019 & 74 & & 3510 & 2164 & 55 & 0003 & 90 \\
\hline & 1680 & 537 & 7 & 0028 & 84 & & 3030 & 1917 & 50 & 0003 & 77 \\
\hline & 1085 & 453 & 9 & 0019 & 39 & & 2900 & 1932 & 47 & 0003 & 74 \\
\hline & 1060 & 453 & 9 & 0020 & 38 & & 2300 & failed & & & \\
\hline & 1025 & 465 & 8 & 0022 & 37 & $Q M-100 R$ & $\infty$ & 720 & & 139 & \\
\hline & 1025 & 474 & 8 & 0024 & 39 & $80-20 / 6$ & 375 & 498 & 38 & 0002 & 123 \\
\hline & 850 & 432 & 8 & 0021 & 29 & $141 \mathrm{~g} / \mathrm{cc}$ & 315 & 423 & 38 & 0002 & 94 \\
\hline & 850 & 438 & 8 & 0022 & 30 & fresh & 250 & fail & & & \\
\hline & 680 & 374 & 8 & 0019 & 21 & unconf & 190 & fail & & & \\
\hline & 650 & 367 & 7 & 0019 & 20 & ref 34 & & & & & \\
\hline & 600 & fail & & & & QM-100R & $\infty$ & 720 & & 146 & \\
\hline ANFO & $\infty$ & 450 & & 64 & & $80-20 / 8$ & 375 & 519 & 35 & 0002 & 128 \\
\hline 095 & 4921 & 443 & 7 & 0053 & 439 & $145 \mathrm{~g} / \mathrm{cc}$ & 315 & 470 & 34 & 0002 & 97 \\
\hline $\mathrm{g} / \mathrm{cc}$ & 4279 & 439 & 8 & 0044 & 325 & fresh & 250 & fail & & & \\
\hline ref 33 & 4424 & 427 & 13 & 0025 & 262 & unconf & 190 & fail & & & \\
\hline & 3937 & 427 & 12 & 0028 & 230 & ref 34 & & & & & \\
\hline & 3679 & 430 & 10 & 0033 & 227 & QM-100R & $\infty$ & 627 & & 154 & \\
\hline & 3515 & 416 & 14 & 0023 & 184 & $70-30 / 6$ & 375 & 605 & 9 & 0009 & 251 \\
\hline & 3125 & 406 & 15 & 0022 & 151 & $144 \mathrm{~g} / \mathrm{cc}$ & 315 & S 91 & 10 & 0008 & 179 \\
\hline & 3005 & 413 & 12 & 0026 & 153 & fresh & 250 & 591 & 8 & 0010 & 142 \\
\hline & 2660 & 408 & 12 & 0026 & 127 & unconf & 190 & 577 & 8 & 0010 & 98 \\
\hline & 2401 & 397 & 13 & 0024 & 108 & ref 34 & 160 & fail & & & \\
\hline & 2117 & 384 & 13 & 0023 & 90 & & 125 & fail & & & \\
\hline & 1911 & 376 & 13 & 0023 & 77 & QM-100R & $\infty$ & 760 & & 165 & \\
\hline & 1640 & 371 & 11 & 0026 & 64 & $60-40 / 8$ & 750 & 726 & 21 & 0004 & 463 \\
\hline & 1447 & 347 & 12 & 0023 & 52 & $146 \mathrm{~g} / \mathrm{cc}$ & 625 & 722 & 19 & 0005 & 374 \\
\hline & 1321 & 3406 & 11 & 0023 & 46 & fresh & 500 & 706 & 19 & 0004 & 265 \\
\hline & 1215 & 3241 & 11 & 0022 & 42 & unconf & 375 & 706 & 14 & 0006 & 200 \\
\hline ANFO & $\infty$ & 6464 & & 95 & & ref 34 & 315 & 668 & 17 & 0005 & 139 \\
\hline NM & 10135 & 6156 & 29 & 0007 & 619 & & 250 & 635 & 17 & 0005 & 100 \\
\hline 1248 & 5115 & 5835 & 24 & 0009 & 247 & & 190 & 620 & 14 & 0005 & 75 \\
\hline $\mathrm{g} / \mathrm{cc}$ & 3895 & 5575 & 23 & 0009 & 169 & & 160 & 605 & 12 & 0006 & 60 \\
\hline unconf & 2625 & 5275 & 19 & 0010 & 102 & & 125 & 552 & 12 & 0006 & 43 \\
\hline ref 12 & 2045 & 4862 & 18 & 0010 & 71 & & 95 & fail & & & \\
\hline & 1595 & 4044 & 18 & 0008 & 49 & & & & & & \\
\hline RX-HD & $\infty$ & 55 & & 125 & & & & & & & \\
\hline 134 & 8438 & 52 & 27 & 0004 & 486 & & & & & & \\
\hline $\mathrm{g} / \mathrm{cc}$ & 8438 & 4 & 78 & 0001 & 285 & & & & & & \\
\hline 31 & 6250 & 44 & 47 & 0002 & 238 & & & & & & \\
\hline ref 1,31 & 5080 & fai & & & & & & & & & \\
\hline
\end{tabular}


Table 2b, page 3

COMPOSITES - ANFO

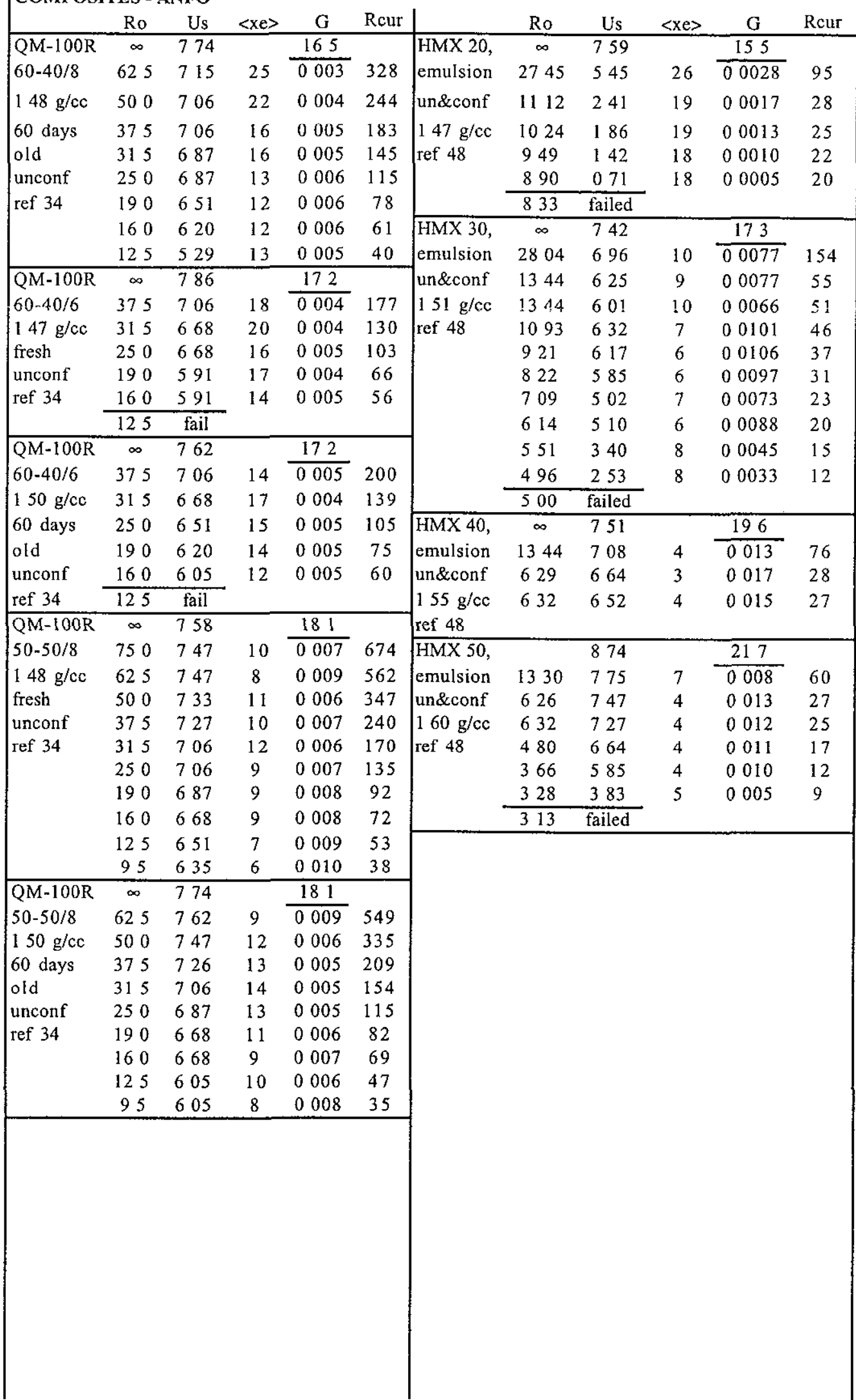




\begin{tabular}{|c|c|c|c|c|c|c|c|c|c|c|c|}
\hline \multicolumn{12}{|c|}{$\begin{array}{l}\text { Table } 2 b \text {, page } 4 \\
\text { COMPOSITES - NON-ALUMINIZED }\end{array}$} \\
\hline & Ro & Us & $<x e>$ & $\mathrm{G}$ & Rcur & & Ro & Us & $\langle\mathrm{xe}\rangle$ & $\mathrm{G}$ & Rcur \\
\hline \multirow{2}{*}{$\begin{array}{l}\text { Amatol } \\
50 / 50\end{array}$} & $\infty$ & 671 & & 184 & & \multirow{2}{*}{$\begin{array}{l}\text { Amatol } \\
50\end{array}$} & $\infty$ & 522 & \multicolumn{2}{|r|}{78} & \multirow[b]{2}{*}{594} \\
\hline & 889 & 635 & 28 & 0002 & 515 & & 123 & 471 & 58 & 0004 & \\
\hline unconf & 635 & 628 & 22 & 0003 & 350 & $10 \mathrm{~g} / \mathrm{cc}$ & 80 & 431 & 55 & 0004 & 310 \\
\hline \multirow{26}{*}{$\begin{array}{l}153 \mathrm{~g} / \mathrm{cc} \\
\text { type } 1 \\
\text { AN un- } \\
\text { sieved, } \\
\text { cast } \\
\text { ref } 30\end{array}$} & 635 & 648 & 15 & 0004 & 430 & \multirow{33}{*}{$\begin{array}{l}\text { TNT 150- } \\
300 \mu \mathrm{m} \\
\text { AN } 840- \\
1200 \mu \mathrm{m} \\
\text { packed, } \\
\text { unconf } \\
\text { ref } 30\end{array}$} & 636 & 418 & 48 & 0005 & 243 \\
\hline & 635 & 630 & 22 & 0003 & 353 & & 636 & 427 & 45 & 0005 & 250 \\
\hline & 635 & 626 & 23 & 0003 & 343 & & 636 & 430 & 44 & 0005 & 250 \\
\hline & 635 & 623 & 24 & 0002 & 339 & & 497 & 415 & 38 & 0006 & 186 \\
\hline & 508 & 646 & 12 & 0005 & 336 & & 497 & 403 & 41 & 0005 & 179 \\
\hline & 508 & 601 & 25 & 0002 & 241 & & 497 & 405 & 40 & 0005 & 181 \\
\hline & 508 & 643 & 14 & 0004 & 321 & & 441 & 388 & 39 & 0005 & 154 \\
\hline & 508 & 654 & 10 & 0006 & 381 & & 441 & 391 & 39 & 0005 & 154 \\
\hline & 508 & 600 & 25 & $0 \cup 02$ & 235 & & 441 & 400 & 37 & 0006 & 158 \\
\hline & 508 & 622 & 19 & 0003 & 272 & & 377 & 375 & 36 & 0006 & 127 \\
\hline & 381 & 627 & 14 & 0004 & 210 & & 377 & 399 & 32 & 0007 & 136 \\
\hline & 381 & 612 & 17 & 0003 & 187 & & 377 & 397 & 32 & 0007 & 132 \\
\hline & 381 & 595 & 20 & 0003 & 176 & & 315 & 382 & 29 & 0007 & 106 \\
\hline & 381 & 581 & 22 & 0003 & 165 & & 315 & 375 & 30 & 0007 & 106 \\
\hline & 381 & 602 & 18 & 0003 & 179 & & 315 & 386 & 28 & 0007 & 106 \\
\hline & 252 & 554 & 17 & 0003 & 99 & & 252 & 372 & 24 & 0008 & 82 \\
\hline & 252 & 546 & 18 & 0003 & 96 & & 252 & 389 & 22 & 0009 & 88 \\
\hline & 252 & 541 & 19 & 0003 & 96 & & 252 & 375 & 24 & 0008 & 85 \\
\hline & 252 & 520 & 21 & 0002 & 91 & & 1915 & 379 & 18 & 0011 & 65 \\
\hline & 252 & 522 & 20 & 0002 & 90 & & 1915 & 358 & 19 & 0010 & 62 \\
\hline & 252 & 559 & 17 & 0003 & 101 & & 1265 & 295 & 16 & 0010 & 37 \\
\hline & 252 & 509 & 21 & 0002 & 88 & & 1265 & 305 & 15 & 0010 & 37 \\
\hline & 252 & 560 & 17 & 0003 & 101 & & 95 & 283 & 12 & 0012 & 28 \\
\hline & 1905 & 489 & 18 & 0003 & 65 & & 95 & 327 & 11 & 0016 & 29 \\
\hline & 1905 & 451 & 20 & 0002 & 61 & & 95 & 273 & 13 & 0011 & 27 \\
\hline & 175 & failed & & & & & 95 & 268 & 13 & 0011 & 27 \\
\hline \multirow{24}{*}{$\begin{array}{l}\text { Amatol } \\
50 / 50 \\
\text { unconf } \\
153 \mathrm{~g} / \mathrm{cc} \\
\text { type } 2 \\
\text { AN } 600- \\
840 \mu \mathrm{m} \text {, } \\
\text { cast } \\
\text { ref } 30\end{array}$} & $\infty$ & 662 & & 184 & & & 8 & 232 & 12 & 0010 & 22 \\
\hline & 9950 & 637 & 25 & 0002 & 643 & & 8 & 241 & 12 & 0011 & 22 \\
\hline & 9950 & 637 & 25 & 0002 & 658 & & 64 & 177 & 11 & 0009 & 16 \\
\hline & 889 & 632 & 25 & 0002 & 543 & & 64 & 187 & 10 & 0009 & 16 \\
\hline & 790 & 628 & 24 & 0002 & 467 & & 64 & 183 & 11 & 0009 & 16 \\
\hline & 790 & 632 & 22 & 0003 & 488 & & 64 & 182 & 11 & 0009 & \multirow[t]{2}{*}{16} \\
\hline & 635 & 630 & 18 & 0003 & 388 & & 625 & \multicolumn{2}{|l|}{ failed } & & \\
\hline & 635 & 635 & 17 & 0004 & 406 & & $\infty$ & 700 & & 177 & \\
\hline & 510 & 625 & 16 & 0004 & 295 & 20 & 508 & 694 & 5 & 0015 & 553 \\
\hline & 510 & 624 & 17 & 0004 & 289 & unconf & 254 & 684 & 4 & 0016 & 198 \\
\hline & 445 & 614 & 17 & 0003 & 235 & $161 \mathrm{~g} / \mathrm{cc}$ & 127 & 653 & 5 & 0015 & 69 \\
\hline & 445 & 616 & 16 & 0004 & 240 & ref 22 & 85 & 603 & 5 & 0012 & 37 \\
\hline & 381 & 607 & 16 & 0004 & 189 & Comp B- & $\infty$ & 775 & & 228 & \\
\hline & 381 & 609 & 16 & 0004 & 196 & AN & 64 & 751 & 14 & 0003 & 450 \\
\hline & 315 & 5847 & 17 & 0003 & 142 & $79-21$ & 64 & 753 & 13 & 0003 & 461 \\
\hline & 315 & 5847 & 17 & 0003 & 142 & $159 \mathrm{~g} / \mathrm{cc}$ & 505 & 754 & 10 & 0005 & 369 \\
\hline & 252 & 5405 & 18 & 0003 & 97 & AN $840-$ & 505 & 751 & 11 & 0.004 & 355 \\
\hline & 252 & 519 & 20 & 0002 & 94 & $1200 \mu \mathrm{m}$ & 378 & 743 & 10 & 0005 & 239 \\
\hline & 252 & 5199 & 20 & 0002 & 94 & unconf & 378 & 757 & 7 & 0007 & 291 \\
\hline & 252 & 5207 & 20 & 0002 & 94 & ref 30 & 378 & 751 & 8 & 0006 & 266 \\
\hline & 252 & 5195 & 20 & 0002 & 94 & & 254 & 755 & 5 & 0009 & 188 \\
\hline & 1905 & 4765 & 18 & 0003 & 65 & & 254 & 776 & & & 298 \\
\hline & 1905 & failed & & & & & 254 & 761 & 4 & 0012 & 212 \\
\hline & 17 & failed & & & & & 254 & 749 & 6 & 0008 & 172 \\
\hline & & & & & & & 254 & 753 & 5 & 0009 & 183 \\
\hline & & & & & & & 191 & 727 & 7 & 0007 & 106 \\
\hline & & & & & & & 191 & 736 & 6 & 0008 & 112 \\
\hline
\end{tabular}




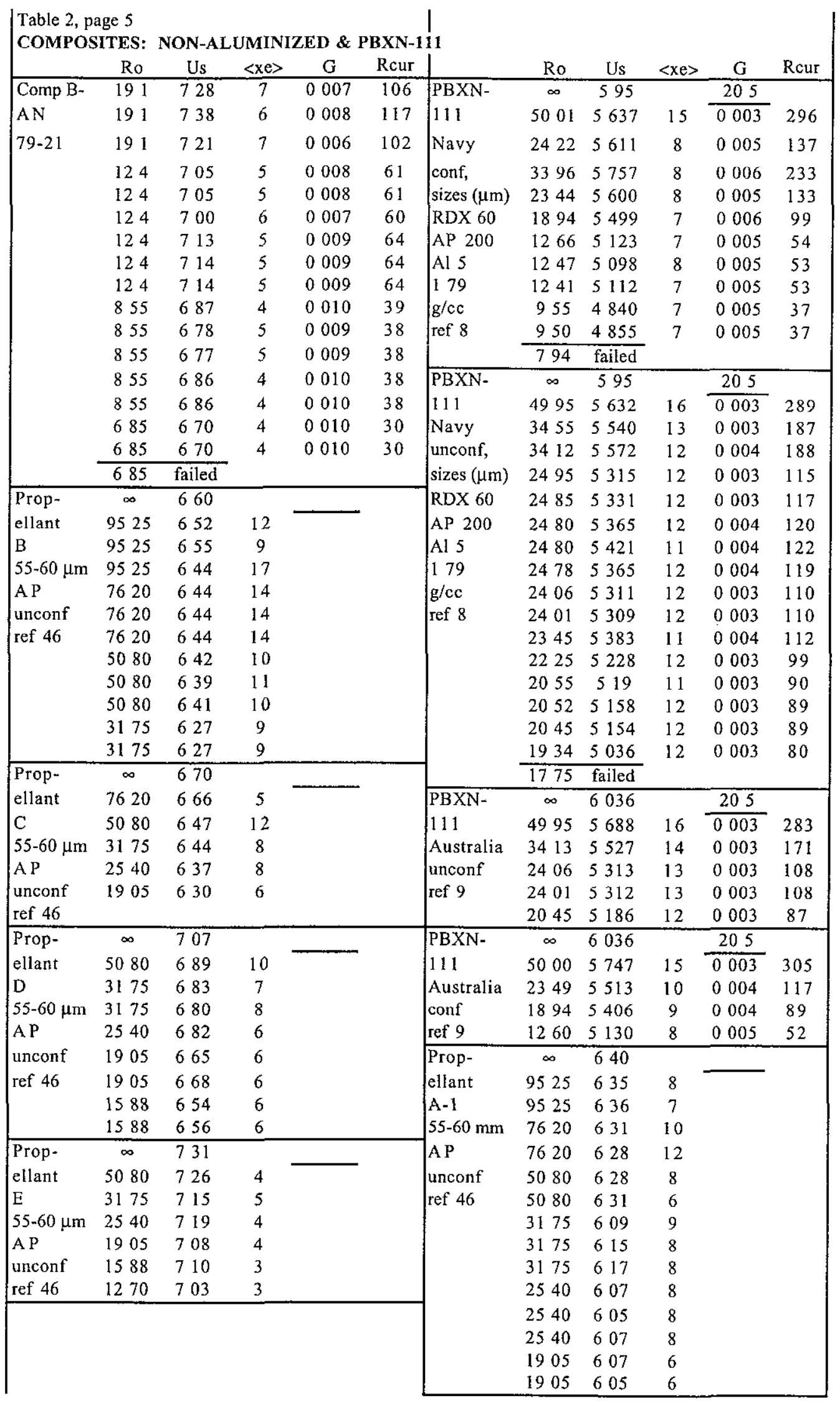




\begin{tabular}{|c|c|c|c|c|c|c|c|c|c|c|c|}
\hline \multicolumn{12}{|c|}{$\begin{array}{l}\text { Table 2, page } 6 \\
\text { COMPOSITES: ALUMINIZED ; INORGANIC }\end{array}$} \\
\hline Prop- & 1905 & 607 & 6 & & & $\mathrm{AP}$ & $\infty$ & 495 & & 5 & \\
\hline ellant & 1588 & 597 & 6 & & & 126 & 381 & 417 & 24 & 002 & 156 \\
\hline$A-1$ & 1588 & 593 & 6 & & & $\mathrm{~g} / \mathrm{cc}$ & 318 & 408 & 22 & 002 & 125 \\
\hline Prop- & $\infty$ & 655 & & & & $10 \mu \mathrm{m}$ & 254 & 385 & 21 & 002 & 90 \\
\hline ellant & 3175 & 633 & 7 & & & ref 45 & 191 & 342 & 19 & 002 & 62 \\
\hline A-2 & 2540 & 635 & 6 & & & & 175 & 333 & 18 & 002 & 55 \\
\hline $10-18 \mu \mathrm{m}$ & 1905 & 634 & 4 & & & AP & $\infty$ & 464 & & 4 & \\
\hline & 1588 & 620 & 5 & & & $124 \mathrm{~g} / \mathrm{cc}$ & 318 & 380 & 22 & 003 & 125 \\
\hline unconf & 1588 & 615 & 5 & & & in steel & 318 & 388 & 21 & 004 & 127 \\
\hline \multirow[t]{2}{*}{ ref 46} & 1270 & 611 & 5 & & & $55-60 \mu \mathrm{m}$ & 127 & 265 & 16 & 003 & 37 \\
\hline & 1270 & 609 & 5 & & & ref 46 & 127 & 265 & 16 & 003 & 37 \\
\hline Prop- & $\infty$ & 596 & & & & & 127 & 265 & 16 & 003 & 37 \\
\hline ellant & 5080 & 579 & 11 & & & $95 \%$ AP & $\infty$ & 339 & & 4 & \\
\hline $\mathrm{F}$ & 3175 & 567 & 9 & & & $5 \% \mathrm{Al}$ & 3810 & 320 & 12 & 005 & 221 \\
\hline $55-60 \mu \mathrm{m}$ & 3175 & 574 & 8 & & & $081 \mathrm{~g} / \mathrm{cc}$ & 2540 & 312 & 10 & 006 & 131 \\
\hline & 2540 & 563 & 8 & & & unconf & 1735 & 298 & 9 & 006 & 78 \\
\hline unconf & 2540 & 565 & 8 & & & ref. 47 & 1270 & 283 & 8 & 007 & 51 \\
\hline \multirow[t]{2}{*}{ ref 46} & 2223 & 560 & 8 & & & & 955 & 266 & 8 & 007 & 36 \\
\hline & 2223 & 555 & 8 & & & $\overline{\mathrm{AP}-\mathrm{Al}}$ & $\infty$ & 433 & & 5 & \\
\hline Prop- & $\infty$ & 464 & & & & ref 47 & 3810 & 401 & 15 & 003 & 200 \\
\hline ellant & 3175 & 380 & 22 & & & $111 \mathrm{~g} / \mathrm{cc}$ & 2540 & 392 & 12 & 004 & 122 \\
\hline$A-1$ & 3175 & 388 & 21 & & & unconf & 1735 & 371 & 10 & 005 & 73 \\
\hline $55-60 \mathrm{~mm}$ & 1270 & 265 & 16 & & & AP $<10$ & 1270 & 355 & 9 & 005 & 50 \\
\hline A P & 1270 & 265 & 16 & & & $\mu \mathrm{m} ; \mathrm{Al}$ & 1270 & 349 & 9 & 005 & 48 \\
\hline \multirow{2}{*}{$\begin{array}{l}\text { conf } \\
\text { ref } 46\end{array}$} & 1270 & 265 & 16 & & & $<7 \mu \mathrm{m}$ & 955 & 315 & 9 & 005 & 33 \\
\hline & & & & & & AP-Al & $\infty$ & 500 & & 6 & \\
\hline $\mathrm{AN}$ & $\infty$ & 445 & & 4 & & ref 47 & 3810 & 452 & 18 & 002 & 182 \\
\hline 10 & 2300 & 282 & 259 & 00022 & 708 & $125 \mathrm{~g} / \mathrm{cc}$ & 2540 & 434 & 14 & 003 & 110 \\
\hline $\mathrm{g} / \mathrm{cc}$ & 2300 & 271 & 271 & 00020 & 688 & unconf & 1735 & 401 & 13 & 003 & 67 \\
\hline unconf & 2020 & 242 & 263 & 00018 & 587 & & 1270 & 362 & 12 & 003 & 43 \\
\hline $300-$ & 2020 & 230 & 274 & 00017 & 571 & $\mathrm{AP}-\mathrm{Al}$ & $\infty$ & 520 & & 6 & \\
\hline $420 \mu \mathrm{m}$ & 1785 & 215 & 253 & 00017 & 491 & ref 47 & 3810 & 467 & 18 & 002 & 179 \\
\hline \multirow[t]{11}{*}{ ref 30} & 1270 & 189 & 193 & 00020 & 331 & $133 \mathrm{~g} / \mathrm{cc}$ & 2540 & 441 & 16 & 002 & 104 \\
\hline & 1270 & 160 & 208 & 00015 & 331 & unconf & 1735 & 404 & 14 & 003 & 63 \\
\hline & 1270 & 161 & 207 & 00016 & 331 & Baratol & $\infty$ & 4896 & & 6 & \\
\hline & 995 & 163 & 161 & 00020 & 259 & 76 & 5080 & 4767 & 10 & 004 & 376 \\
\hline & 995 & 167 & 160 & 00021 & 259 & $26 \mathrm{~g} / \mathrm{cc}$ & 3265 & 4700 & 8 & 005 & 209 \\
\hline & 995 & 136 & 172 & 00016 & 246 & unconf & 2407 & 4625 & 8 & 005 & 139 \\
\hline & 870 & 155 & 144 & 00021 & 215 & ref 22 & 1904 & failed & & & \\
\hline & 870 & 135 & 150 & 00018 & 215 & & & & & & \\
\hline & 800 & 146 & 135 & 00022 & 198 & & & & & & \\
\hline & 800 & 115 & 144 & 00016 & 189 & & & & & & \\
\hline & 636 & failed & & & & & & & & & \\
\hline AP & $\infty$ & 239 & & 25 & & & & & & & \\
\hline $061 \mathrm{~g} / \mathrm{cc}$ & 254 & 204 & 16 & 007 & 106 & & & & & & \\
\hline $\begin{array}{l}10 \mu \mathrm{m} \\
\text { ref } 45\end{array}$ & 127 & 169 & 12 & 007 & 42 & & & & & & \\
\hline AP & $\infty$ & 373 & & 4 & & & & & & & \\
\hline $10 \mathrm{~g} / \mathrm{cc}$ & 1220 & 360 & 29 & 002 & 817 & & & & & & \\
\hline $13 \mu \mathrm{m}$ & 510 & 339 & 23 & 003 & 251 & & & & & & \\
\hline ref 44 & 175 & 278 & 15 & 004 & 61 & & & & & & \\
\hline $\mathrm{AP}$ & $\infty$ & 374 & & 4 & & & & & & & \\
\hline $10 \mathrm{~g} / \mathrm{cc}$ & 381 & 343 & 16 & 004 & 189 & & & & & & \\
\hline $10 \mu \mathrm{m}$ & 254 & 328 & 14 & 005 & 112 & & & & & & \\
\hline ref 45 & 175 & 305 & 12 & 005 & 68 & & & ' & & & \\
\hline & 127 & 282 & 11 & 005 & 45 & & & & & & \\
\hline
\end{tabular}


Table 3 Data for cylinders where failure was measured

\begin{tabular}{|c|c|c|c|c|c|c|c|c|}
\hline & \multirow{2}{*}{$\begin{array}{c}\rho_{o} \\
(g / c c)\end{array}$} & \multirow{2}{*}{$\begin{array}{c}\mathbf{R}_{\mathbf{r}} \\
(\mathrm{mm})\end{array}$} & \multicolumn{2}{|c|}{ detvel $(\mathrm{mm} / \mu \mathrm{s})$} & \multirow{2}{*}{$\begin{array}{l}\left\langle\mathrm{x}_{\mathrm{e}}\right\rangle_{\mathrm{f}} \\
(\mathrm{mm})\end{array}$} & \multirow{2}{*}{$\begin{array}{l}\mathrm{G}\left(\mu \mathrm{s}^{-1}\right. \\
\left.{ }^{*} \mathrm{GPa}^{-2}\right)\end{array}$} & \multirow{2}{*}{$\begin{array}{l}R_{\text {cur }} \\
(\mathrm{mm})\end{array}$} & \multirow[b]{2}{*}{ ref } \\
\hline & & & $\overline{\mathrm{U}_{\mathrm{sf}}}$ & $\mathrm{D}$ & & & & \\
\hline \multicolumn{9}{|l|}{ Homogeneous } \\
\hline TNT, pressed & 080 & 375 & 18 & 504 & 65 & 0040 & 23 & 37 \\
\hline TNT, pressed & 100 & 425 & 34 & 523 & 45 & 0049 & 43 & 37 \\
\hline TNT, pressed & 146 & 221 & 57 & 670 & 14 & 0062 & 35 & 37 \\
\hline TNT, pressed & 162 & 131 & 66 & 708 & 06 & 0084 & 29 & 37 \\
\hline TNT, cast & 162 & 725 & 66 & 713 & 28 & 0018 & 153 & 37 \\
\hline TNT, creamed & 162 & 73 & & 694 & & & & 27 \\
\hline TNT, liquid & 144 & 142 & & 657 & 3 & & & 27 \\
\hline ietryl & 094 & 392 & 20 & 586 & 8 & 0013 & 24 & 38 \\
\hline tetryl & 098 & 238 & 48 & 585 & 2 & 011 & 34 & 38 \\
\hline NM & 103 & 136 & 612 & 621 & 02 & 081 & 53 & 22 \\
\hline NTO & 175 & 8 & 76 & 778 & 1 & 0039 & 26 & 25 \\
\hline NTO & 185 & 85 & 79 & 823 & 2 & 0015 & 23 & 25 \\
\hline NTO & 187 & 127 & 81 & 825 & 2 & 0016 & 46 & 24 \\
\hline \multicolumn{9}{|l|}{ Heterogeneous } \\
\hline XTX-8003 & 153 & 019 & 705 & 7307 & 004 & 17 & 054 & 22 \\
\hline PBX-9404 & 183 & 060 & 727 & 8805 & 005 & 039 & 09 & 22 \\
\hline NM-silica-guar & 117 & 477 & 492 & 6550 & 4 & 0033 & 6 & 4 \\
\hline$X-0219$ & 191 & 75 & 735 & 768 & 2 & 0017 & 20 & 22 \\
\hline$X-0290$ & 190 & 45 & & 771 & & & 13 & 27 \\
\hline PBX-9502 & 189 & 388 & 742 & 778 & 10 & 0031 & 10 & 26 \\
\hline RDX 94 6/pew & 156 & 25 & 60 & 850 & 28 & 0013 & 3 & 43 \\
\hline RDX $978 /$ pew & 156 & 126 & 54 & 850 & 17 & 0018 & 12 & 43 \\
\hline Comp B & 171 & 214 & 67 & 792 & 13 & 0023 & 3 & 23 \\
\hline Cyclotol 77-23 & 174 & 30 & 76 & 821 & 1 & 0028 & 6 & 22 \\
\hline PBXW-7 & 170 & 128 & 725 & 792 & 06 & 0073 & 3 & 43 \\
\hline \multicolumn{9}{|l|}{ Composites } \\
\hline ANFO, SW & 116 & 625 & 36 & 588 & 7 & 0019 & 6 & 10,11 \\
\hline RX-HD & 134 & 56 & 4 & 55 & 40 & 00020 & 65 & 31 \\
\hline HANFO & 107 & 26 & 19 & 535 & 45 & 00035 & 16 & 9 \\
\hline QM-100R 80-20/6 & 141 & 288 & 42 & 720 & 38 & 00018 & 26 & 34 \\
\hline QM-100R 80-20/8 & 145 & 288 & 47 & 720 & 34 & 00021 & 29 & 34 \\
\hline QM-100R $70-30 / 6$ & 144 & 175 & 57 & 627 & 8 & 00096 & 34 & 34 \\
\hline QM-100R $60-40 / 8$ & 146 & 11 & 55 & 760 & 12 & 00054 & 13 & 34 \\
\hline QM-100R 60-40/6 & 147 & 142 & 59 & 786 & 12 & 00053 & 17 & 34 \\
\hline QM-100R $60-40 / 6$ & 150 & 142 & 60 & 762 & 12 & 00054 & 19 & 34 \\
\hline HMX 20, emulsion & 147 & 86 & 07 & 759 & 18 & 00005 & 3 & 48 \\
\hline HMX 30, emulsion & 151 & 50 & 25 & 742 & 8 & 00033 & 3 & 48 \\
\hline HMX 50, emulsion & 160 & 32 & 38 & 874 & 4 & 00065 & 2 & 48 \\
\hline Amatol $50 / 50$ & 153 & 183 & 45 & 671 & 19 & 00022 & 19 & 30 \\
\hline Amatol $50 / 50$ & 153 & 183 & 47 & 662 & 19 & 00023 & 21 & 30 \\
\hline Amatol $50 / 50$ & 100 & 632 & 18 & 522 & 10 & 00095 & 4 & 30 \\
\hline Amatex 20 & 161 & 85 & & 703 & 10 & & 2 & 27 \\
\hline CompB-AN 79-21 & 159 & 685 & 67 & 775 & 4 & 0010 & 11 & 30 \\
\hline PBXN-111, N, conf & 179 & 87 & 48 & 595 & 7 & 00052 & 12 & 8 \\
\hline PBXN-111, N, unc & 179 & 185 & 50 & 595 & 12 & 00032 & 28 & 8 \\
\hline Baratol & 260 & 215 & 46 & 490 & 8 & 0051 & 49 & 22 \\
\hline AN & 100 & 72 & 11 & 445 & 130 & 0002 & 37 & 30 \\
\hline
\end{tabular}




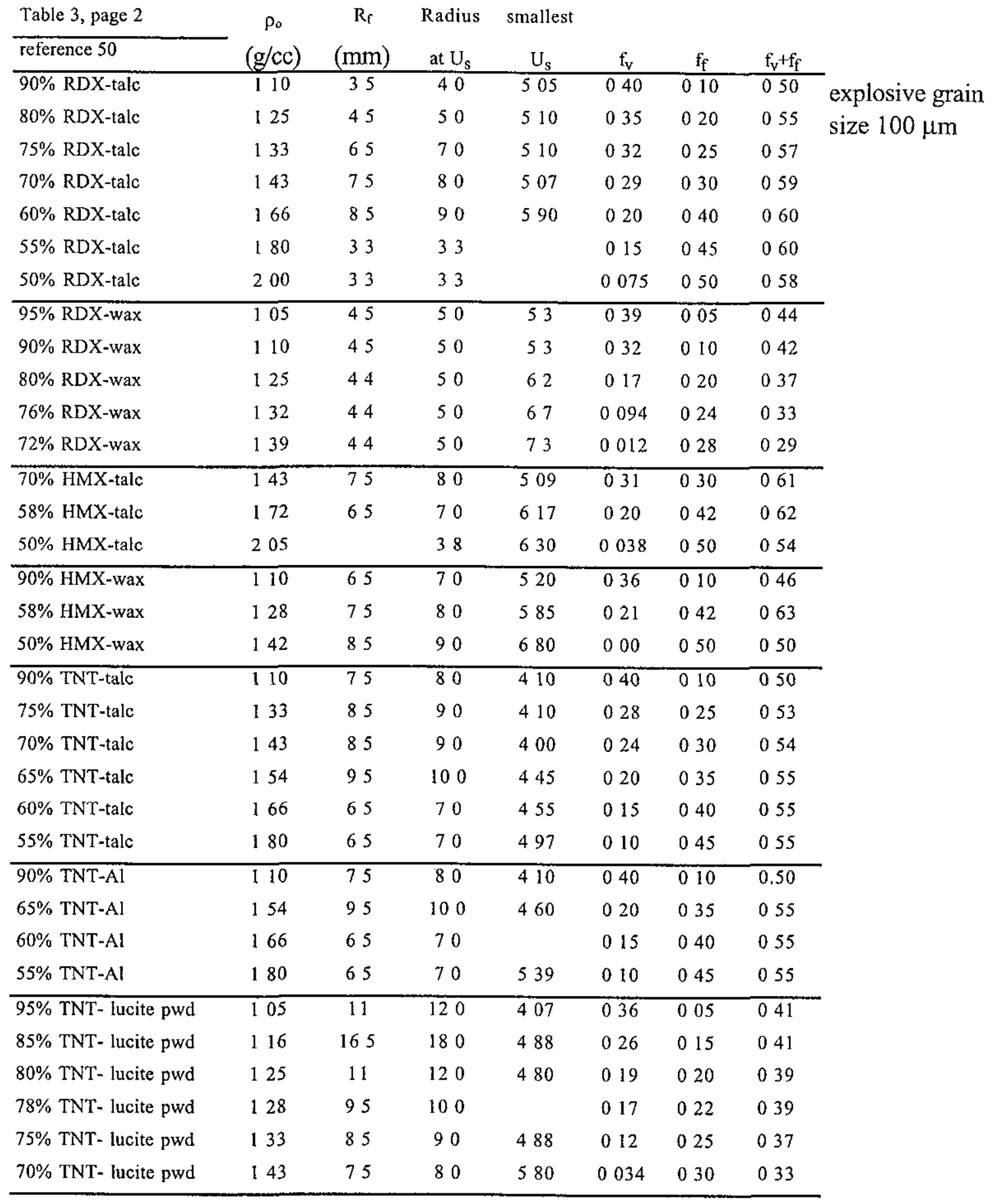


Table 3 , page 3

\begin{tabular}{lccl}
\hline reference 51 & $\rho_{0}$ & $R_{f}$ & containment \\
\hline Liquid TNT, 850C & 146 & 38 & $13 \mathrm{~mm}$ steel \\
& & 60 & glass \\
& & 90 & cellophane \\
\hline Liquid TNT, 950C & 145 & 36 & $13 \mathrm{~mm}$ steel \\
& & 48 & glass \\
& & 72 & cellophane \\
\hline $92 \%$ NM, acetone & 110 & 35 & $13 \mathrm{~mm}$ steel \\
& & 27 & glass \\
& & 42 & cellophane \\
\hline $90 \%$ NM, acetone & 109 & 46 & 2 -12 mm steel \\
& & 33 & glass \\
& & 54 & cellophane \\
\hline $813 \%$ NM, acetone & 105 & 17 & $3-12$ mm steel \\
& & 136 & glass \\
& & 220 & cellophane \\
\hline AN & 106 & $<3$ & 5 mm steel \\
& & $>80$ & glass \\
& & $>100$ & cellophane \\
\hline
\end{tabular}


Table 4 Compositions of explosives used in this report

\begin{tabular}{|c|c|}
\hline Explosive & Composition of Mixtures (wt\%) \\
\hline ABNBF & 7-amino-4,6-dinitrobenzofuroxan \\
\hline Amatex 20 & AN 80, TNT 20 \\
\hline Amatol $50 / 50$ & AN 50, TNT 50 \\
\hline AN & ammonium nitrate \\
\hline ANFO-generic & AN 94, fuel oil 6 \\
\hline ANFO-NM & AN 77 , water 16, mineral oil 6, emulsifier 1 \\
\hline ANFO-SW & AN 77 , water 147 , oil 39 , sorbitol monooleate 07 , ? \\
\hline AP & ammonium perchlorate \\
\hline $\mathrm{BX}-4$ & TATB 60, RDX $30, \mathrm{HMX}, 5$, Viton A 5 \\
\hline $\mathrm{C}-4$ & RDX 91, pib 9 \\
\hline Comp A & RDX 91, wax 9 \\
\hline Comp B & RDX 63, TNT 36, Wax 1 \\
\hline Cyclotol & RDX 75 , TNT 25 \\
\hline Cyclotol 77-23 & RDX 77, TNT 23 \\
\hline DINGU & dinitroglycolurile \\
\hline DNBF & 4,6-dinitrobenzofuroxan \\
\hline DNT & 2,4-dinitrotoluene \\
\hline EDC-35 & TATB 95, kel-F 5 (moist-aminated TATB) \\
\hline EDNA & ethylenediamine nitramine \\
\hline HANFO & AN 72 4, fuel oil 46, AN/water/emulsion 23 \\
\hline HMX 20-emulsion & AN 572 , HMX 20, SN 80 , water 96 , fuel oil 52 \\
\hline HMX 30-emulsion & AN $501, \mathrm{HMX} 30$, SN 70 , water 84 , fuel oil 46 \\
\hline HMX 40-emulsion & AN 429 , HMX 40 , SN 60 , water 72 , fuel oil 39 \\
\hline HMX 50-emulsion & HMX 50, AN 358 , SN 50 , water 60 , fuel oil 33 \\
\hline IRX-1 & HMX 70, htpb 30 \\
\hline IRX-3A & HMX 70, htpb 20, Al 10 \\
\hline IRX-4 & HMX 30, htpb 30, AP 24, Al 16 \\
\hline LX-04 & HMX 85, Viton-A 15 \\
\hline LX-10 & HMX 90, Viton-A 10 \\
\hline LX-17-1 & TATB 925 , kel-F 75 (wet-aminated TATB) \\
\hline NM & NM 94, nitroethane 4, nitropropane 1 \\
\hline NM-silica & NM 93, silica 6, guar gum 1 (gel) \\
\hline NTO & 5-nitro-1,2,4-triazol-2-one \\
\hline Octol & HMX 75, TNT 25 \\
\hline PBX-9404 & HMX 94, nitrocellulose-113, c e phosphate 3 \\
\hline PBX-9501 & HMX 95 , estane 25 , bdnpa 125 , bdnpf 125 \\
\hline PBX-9502 & TATB 95, kel-F 5 (dry-aminated TATB) \\
\hline PBXN-110 & HMX 88, htpb 12 \\
\hline PBXN-111 & $\mathrm{RDX} 20, \mathrm{AP} 43, \mathrm{Al} 25$, htpb 12 \\
\hline PBXW-7 type II & TATB 60, RDX 35, Viton A 5 \\
\hline PBXW-123 & AP 448 , Al 302 , TMETN 188 , pcl 42 , ndpa 1, ipdi 1 \\
\hline PBXW-126 & Al 26, NTO 22, AP 20, RDX 20, polyurethane 12 \\
\hline Pentolite & PETN 50, TNT 50 \\
\hline Picric Acid & 2,4,6-trinitrophenol \\
\hline Propellant A & plastisol-NC 55, AP 30, Al 15 \\
\hline Propellant B & plastisol-NC 647 , AP 353 \\
\hline
\end{tabular}


Table 3, page 2

\begin{tabular}{|c|c|}
\hline Explosive & Composition of Mixtures (wt\%) \\
\hline Propellant C & plastisol-NC 64 7, AP 29 4, RDX 59 \\
\hline Propellant D & plastisol-NC 647, AP20 3, RDX 15 \\
\hline Propellant E & plastisol-NC 647 , RDX 353 \\
\hline Propellant $F$ & 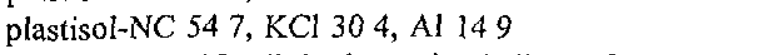 \\
\hline QM-100 & AN 75 , water 18 , oil 5 , glass microballoons 2 \\
\hline QM-100R, 80-20/6 & AN 604 , RDX 20, water 151 , fuel oil 45 \\
\hline QM-100R, 80-20/8 & AN 593 , RDX 20, water 148 , fuel oil 59 \\
\hline QM-100R, 70-30/6 & AN $528, \operatorname{RDX} 30$, water 132 , fuel oil 40 \\
\hline QM-100R, 60-40/6 & AN $453, \operatorname{RDX} 40$, water 113 , fuel oil 34 \\
\hline QM-100R, $60-40 / 8$ & AN $444, \operatorname{RDX} 40$, water 111 , fuel oil 44 \\
\hline QM-100R, 50-50/8 & AN 370, RDX 50, water 93 , fuel oil 37 \\
\hline RDX-pew & RDX variable, polyethylene wax variable \\
\hline $70 \% \mathrm{RDX}$-urethane & RDX 70, polyurethane 2965 , graphite 035 \\
\hline $72 \mathrm{RDX}$-urethane $\# 3$ & RDX 72, binder 28 C 33\%,H 60, O 5 4, $097 \mathrm{~g} / \mathrm{cc}$ \\
\hline 72 RDX-urethane\#5 & RDX 72, binder $28 \mathrm{C} 14 \%, \mathrm{H} 29, \mathrm{O} 29$, Si $29,102 \mathrm{~g} / \mathrm{cc}$ \\
\hline RX-08-HD & HMX 74, TMETN 20, Tone 26052 \\
\hline $\mathrm{RX}-52-\mathrm{AD}$ & TATB 65, FEFO 31, pcl 16, pvf $14, n-10011$ \\
\hline $\mathrm{RX}-52-\mathrm{AE}$ & TATB 65, FEFO, 32, pcl, 16, pvf, 14 \\
\hline $\mathrm{RX}-54-\mathrm{AJ}$ & HMX 47, Al 28, TMETN $16, \mathrm{NC} 8$ \\
\hline RX-HD & AN 787 , fuel oil 64 , calcium nitrate 55 , water 94 \\
\hline $\mathrm{T} 2$ & TATB 97, unknown binder 3 \\
\hline Tetryl & trinitro-2,4,6-phenylmethylnitramine \\
\hline Ult TATB & ultrafine-grain $(6 \mu \mathrm{m})$ TATB \\
\hline$X-0219$ & TATB 90, Kel-F 10 \\
\hline $\mathrm{X}-0290$ & TATB 95, Kel-F 5, became PBX-9502 \\
\hline $\mathrm{X}-0341$ & TATB 9025, HMX 475 , kel-F 5 \\
\hline $\mathrm{X}-0342$ & TATB 85 5, HMX 95, kel-F 5 \\
\hline $\mathrm{X}-0343$ & TATB 8075 , HMX 1425 , kel-F 5 \\
\hline $\mathrm{X}-0344$ & TATB 71 25, HMX 23 75, kel-F 5 \\
\hline XTX-8003 & PETN 80, silicone rubber (sylgard) 20 \\
\hline Binder & Composition \\
\hline htpb & hydroxy-terminated polybutadiene \\
\hline idpi & isophorone diisocyanate \\
\hline$n-100$ & commerical isoceprate \\
\hline ndpa & nitrodiphenylamine \\
\hline $\mathrm{pcl}$ & polycaprolactone \\
\hline pew & polyethylene wax \\
\hline pib & polyisobutylene \\
\hline $\begin{array}{l}\text { plastisol-NC } \\
\text { pvf }\end{array}$ & $\begin{array}{l}\text { nitrocellulose with about } 10 \% \text { nitrogylcerin } \\
\text { polyvinylchloride }\end{array}$ \\
\hline \multicolumn{2}{|l|}{ Abbreviations } \\
\hline ANFO-NM & from New Mexico (Lee) \\
\hline ANFO-SW & from Sweden (Swedish Rock) \\
\hline PBXN-111-N & from U S Navy (Forbes) \\
\hline PBXN-111-A & from Australia (Kennedy) \\
\hline
\end{tabular}




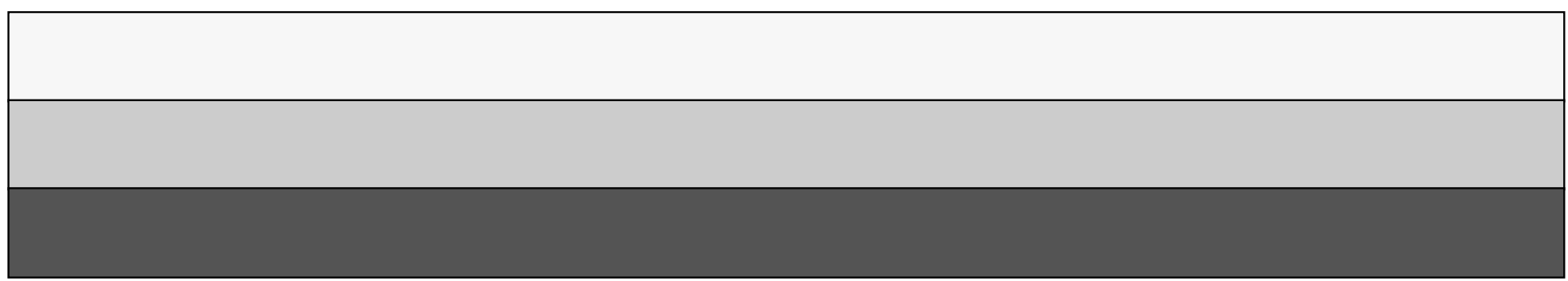

